\title{
Binary blazed reflection grating for UV/VIS/NIR/SWIR spectral range
}

Thomas Flügel-Paul $^{\text {a }}$

Frank Burmeister ${ }^{\mathrm{a}}$

Uwe D. Zeitnera
Mane-Si-Laure L. Bouhours ${ }^{b}$

Gaëlle Lehoucq ${ }^{b}$

Julie Cholet ${ }^{b}$

Brigitte Loiseaux ${ }^{b}$

Nicolas Tetaz ${ }^{\mathrm{C}}$
Roman Windpassinger ${ }^{d}$

Mattheo Taccolad

aFraunhofer Institute for Applied Optics, Jena, Germany;

${ }^{b}$ Thales Research \& Technology, Palaiseau, France;

'Thales Alenia Space, Cannes la Bocca Cedex, France;

dEuropean Space Agency, ESTEC, Noordwijk, Netherlands 


\section{Hyperspectral Imaging Spectrometry}

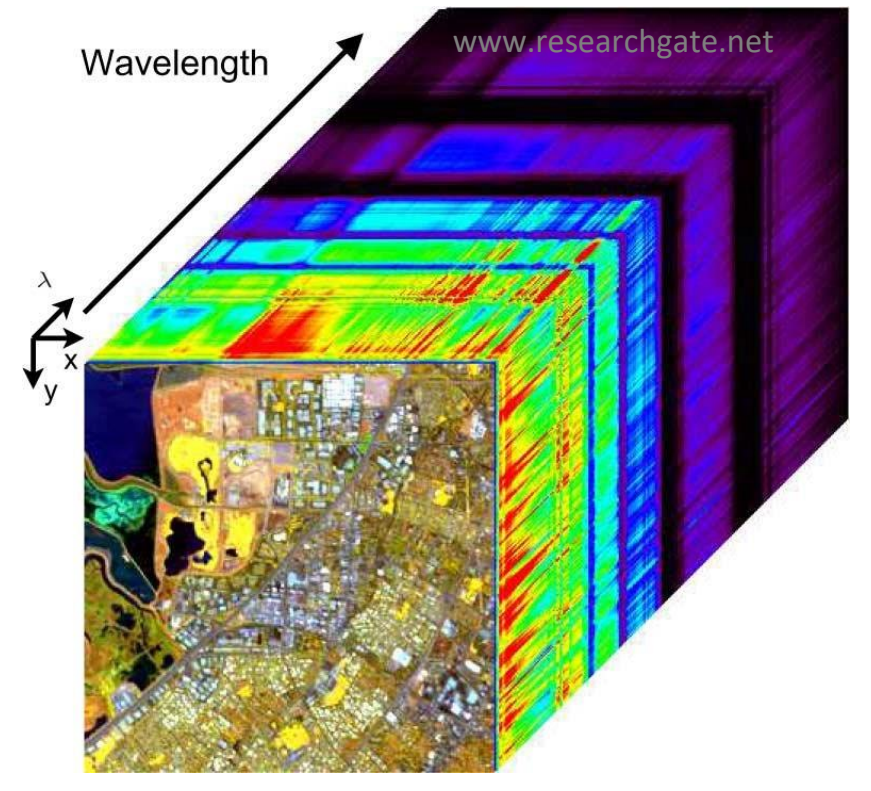

$>$ different surface textures/conditons show characteristic spectra

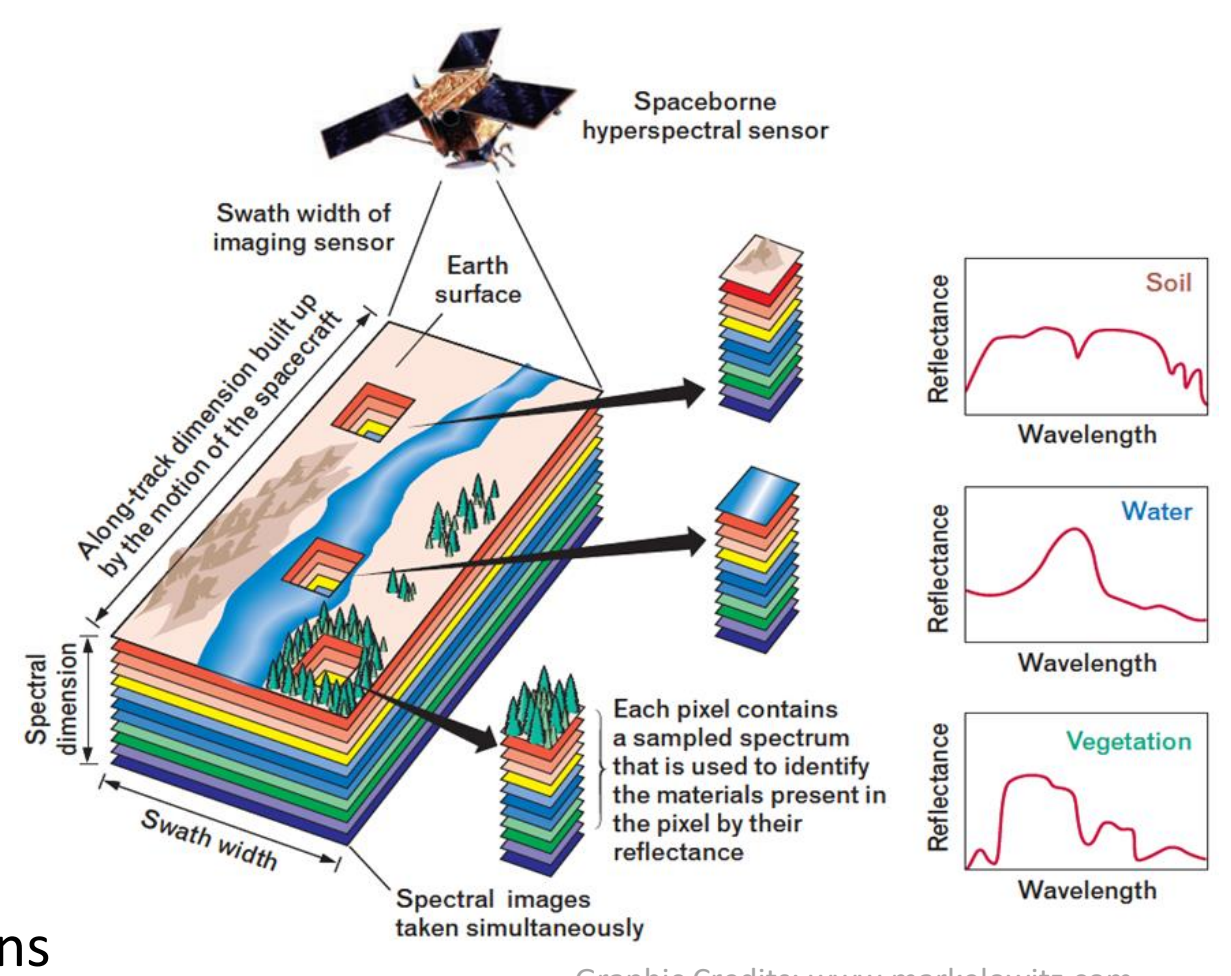

Graphic Credits: www.markelowitz.com

$>$ application in mining, forestry, agriculture, ... and many more

$>$ rapidely growing commercial market down to handheld spectrometry 


\section{Imaging Spectrometer}

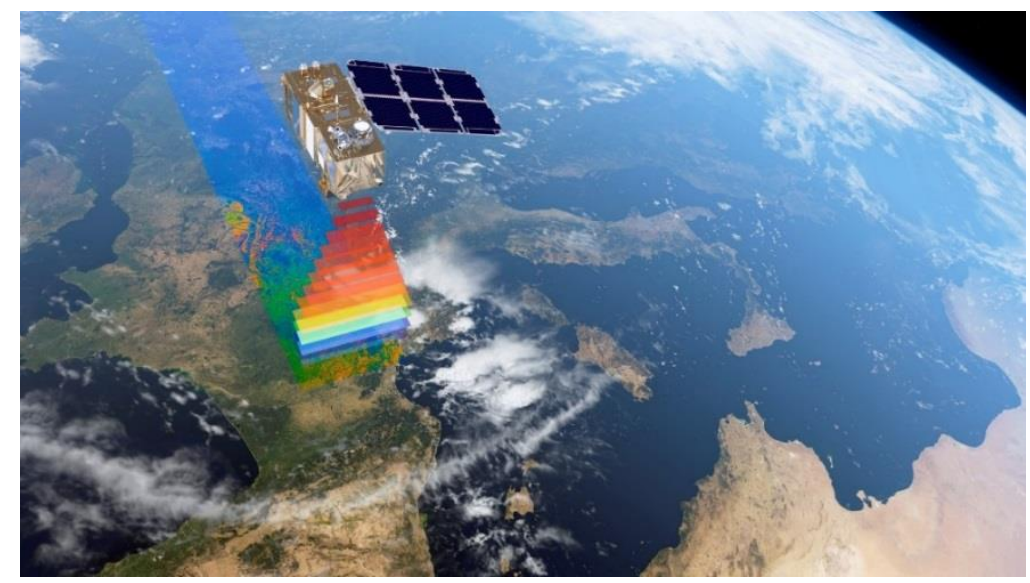

Sentinel 2 colour vision*

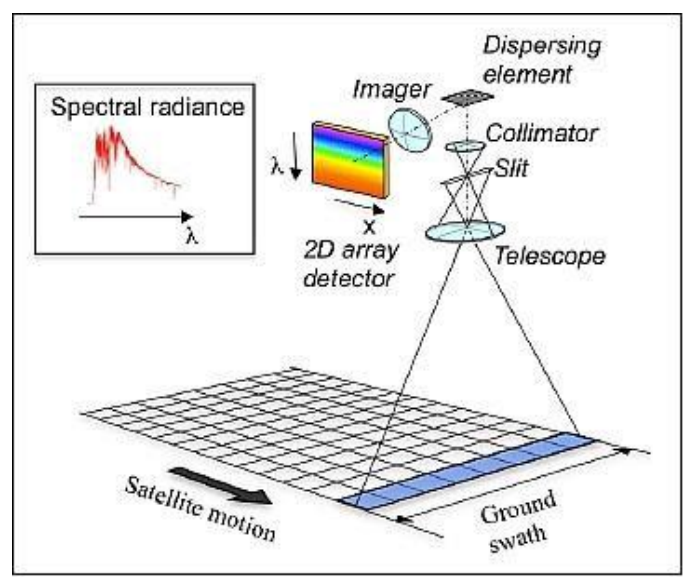

Pushbroom imaging*

> 2014 Technology research program by ESA „Advances large FOV UV/VIS/NIR/SWIR Spectrometers"

$>$ Proposal for imaging spectrometers with advanced properties:

- Compactness

- Increased spectral range and FOV

- $340 \mathrm{~nm}-2500 \mathrm{~nm}$

- or: $340 n m-1050 n m+1000 n m-2500 n m(2$ channels) 


\section{Potential Spectrometer Architecture: Dyson}

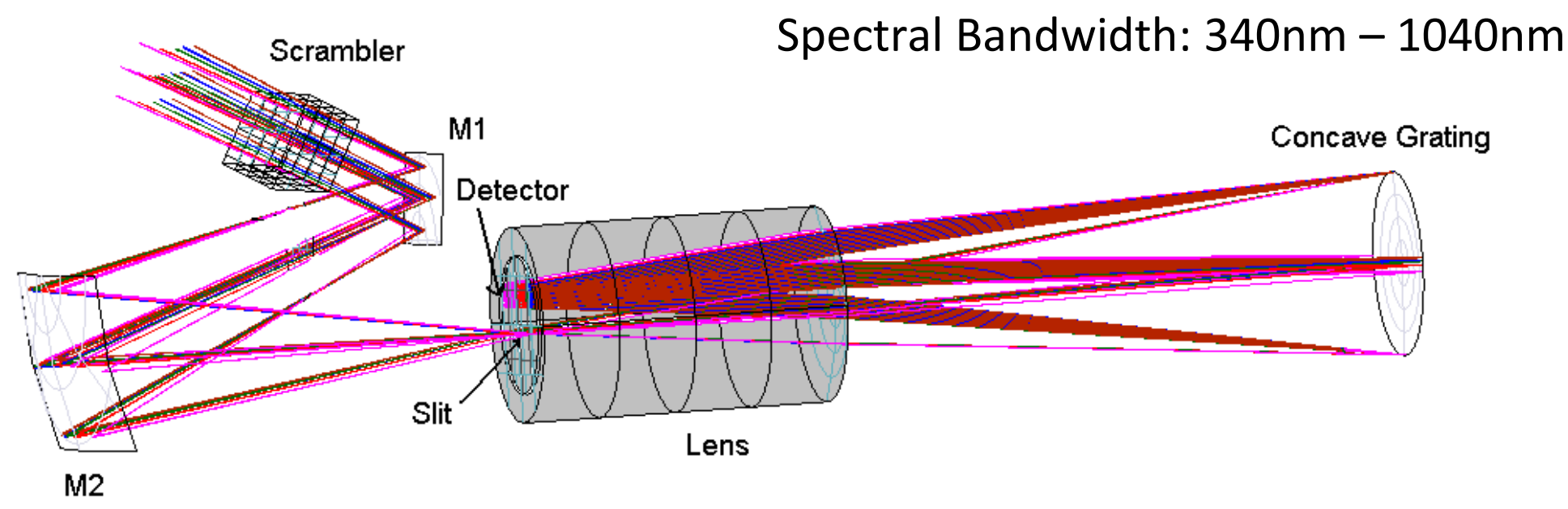




\section{Potential Spectrometer Architecture: Dyson}

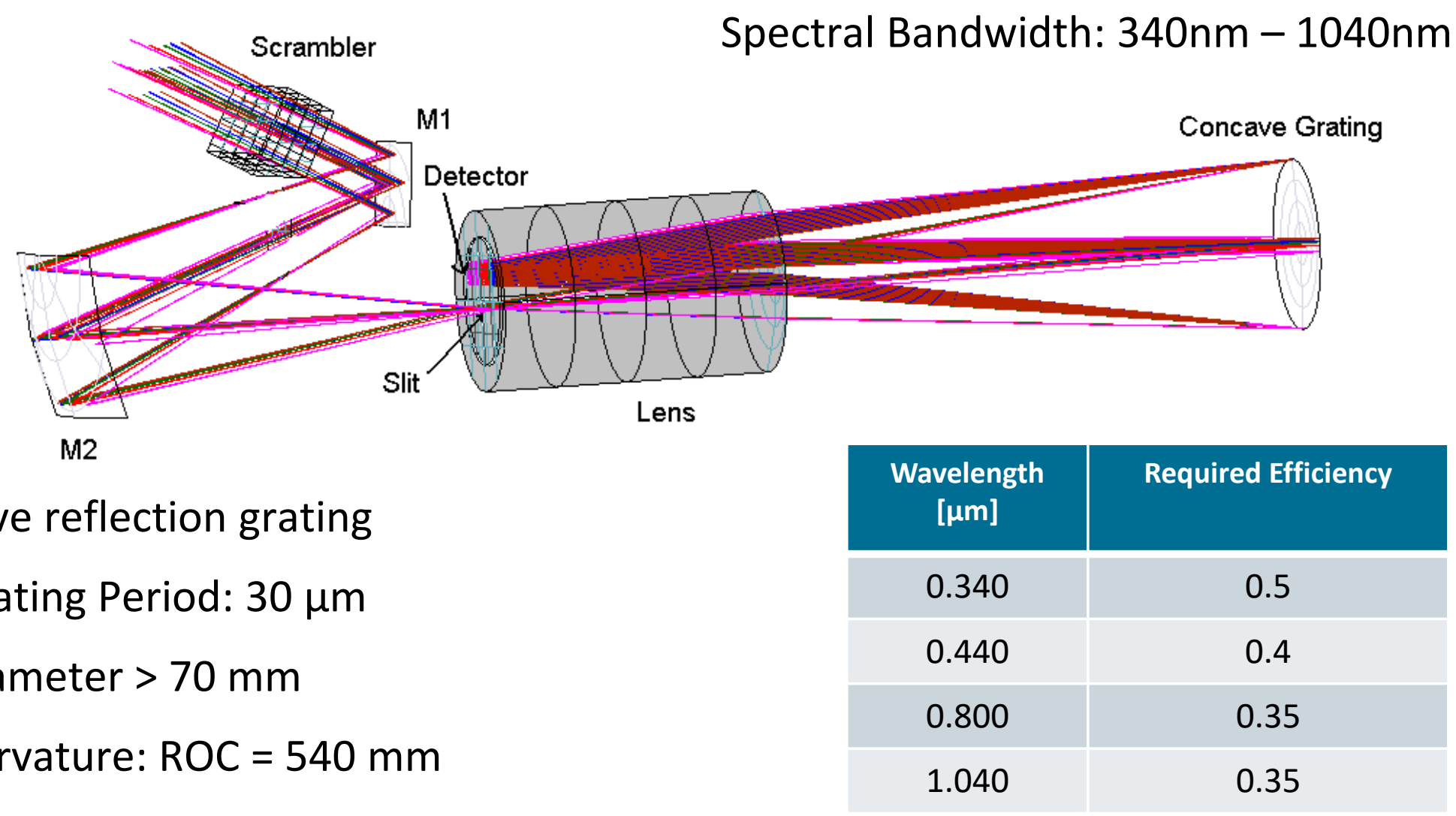

Concave reflection grating

$>$ Grating Period: $30 \mu \mathrm{m}$

$>$ Diameter $>70 \mathrm{~mm}$

$>$ Curvature: $\mathrm{ROC}=540 \mathrm{~mm}$

\begin{tabular}{|c|c|}
\hline $\begin{array}{c}\text { Wavelength } \\
{[\mu \mathrm{m}]}\end{array}$ & Required Efficiency \\
\hline 0.340 & 0.5 \\
\hline 0.440 & 0.4 \\
\hline 0.800 & 0.35 \\
\hline 1.040 & 0.35 \\
\hline
\end{tabular}

Efficiency over such a large spectral band difficult to achieve with classical blazed gratings

Reference: N. Tetaz et al, “Advances large FOV UV/VIS/NIR/SWIR Spectrometers“ International Conference on Space Optics, Tenerife 2014 


\section{Wide Band and High Efficiency Reflective Grating}

$>$ Demonstration of the feasibility of a curved effective medium grating that can meet the requirements of next generation spectrometers.

ThalesAlenia

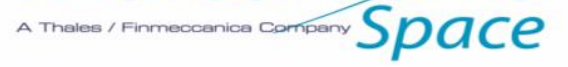
Optical Design $+$ Grating Requirements

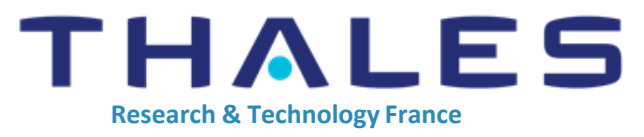

Grating fabrication

by

Nano-Imprint Lithography

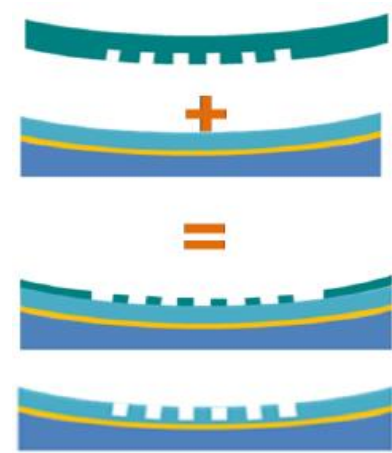

\section{Fraunhofer} IOF

Grating fabrication

by

E-Beam Lithography 


\section{Wide Band and High Efficiency Reflective Grating}

$>$ Demonstration of the feasibility of a curved effective medium grating that can meet the requirements of next generation spectrometers.

\section{ThalesAlenia} Than

Optical Design $+$

Grating Requirements

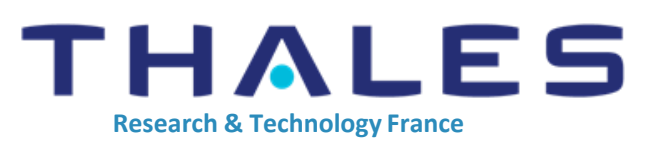

Grating fabrication

by

Nano-Imprint Lithography

\section{Fraunhofer}

IOF

Grating fabrication

by

E-Beam Lithography

\section{Project Phases}

Technology Development on Plane Substrates

Application to Curved Substrates

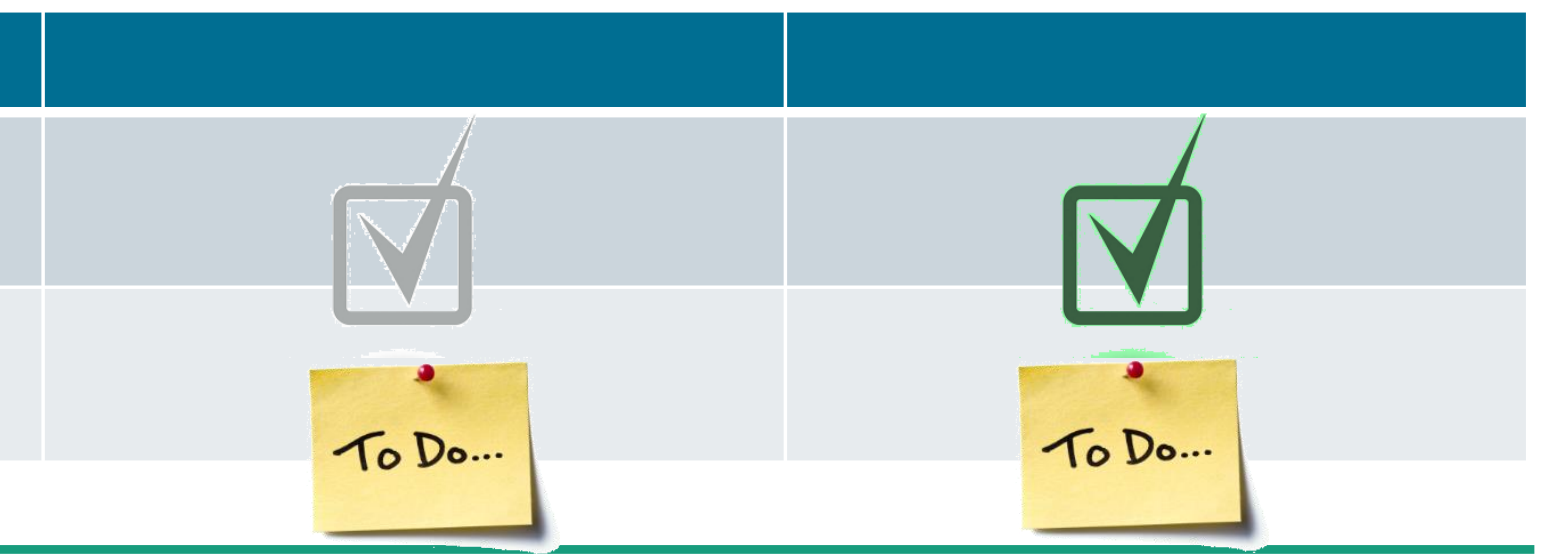




\section{Binary Blazed Effective Medium Grating}

Blazed grating

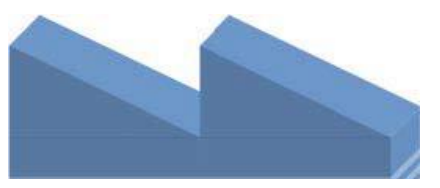

- Optimized for one wavelength

- Large efficiency drop-down for wide spectral range
Effective medium grating

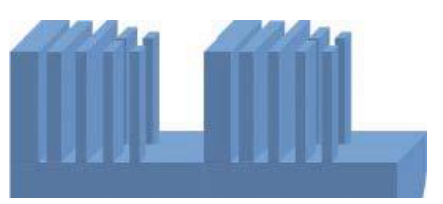

- Subwavelength structures which are not „resolved" by the incident light

- Local refractive index is a function of the filling factor

- feature sizes can be tailored to achieve high efficiency over a wide spectral range. 


\section{Binary Blazed Effective Medium Grating}

Blazed grating

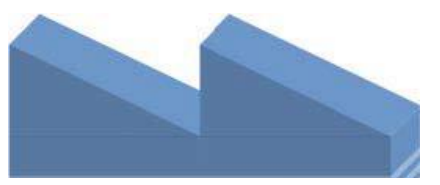

- Optimized for one wavelength

- Large efficiency drop-down for wide spectral range

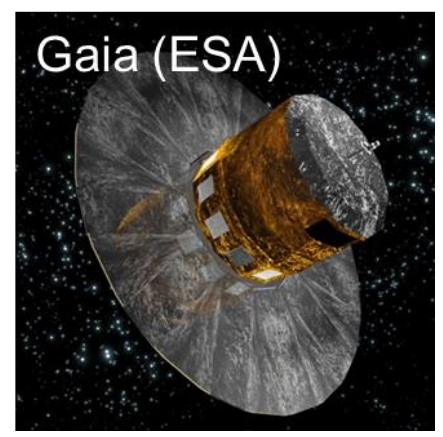

grating period: min. feature size: structure depth:

\section{size:} efficiency:

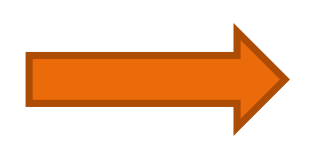

$3.31 \mu \mathrm{m}$

$200 \mathrm{~nm}$

$1.8 \mu \mathrm{m}$

$205 \mathrm{~mm} \times 155 \mathrm{~mm}$

$>80 \%$
Effective medium grating

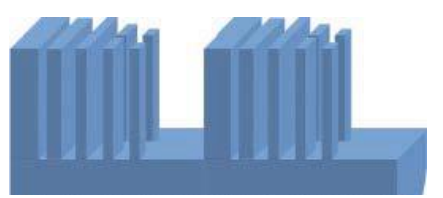

- Subwavelength structures which are not „resolved" by the incident light

- Local refractive index is a function of the filling factor

- feature sizes can be tailored to achieve high efficiency over a wide spectral range.
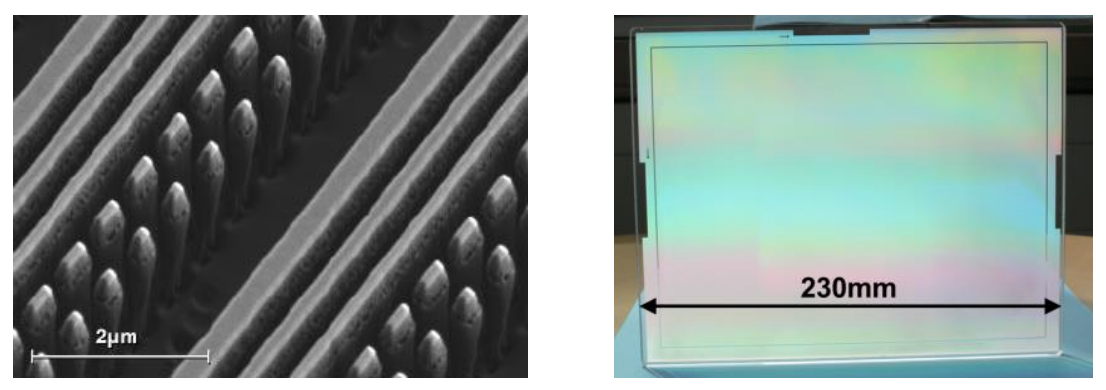


\section{Grating Design Wide Band Grating}

$>$ overall blaze period: $30 \mu \mathrm{m}$

$>$ subfeature displacement: $500 \mathrm{~nm} \times 500 \mathrm{~nm}$

$>$ continuous feature size variation

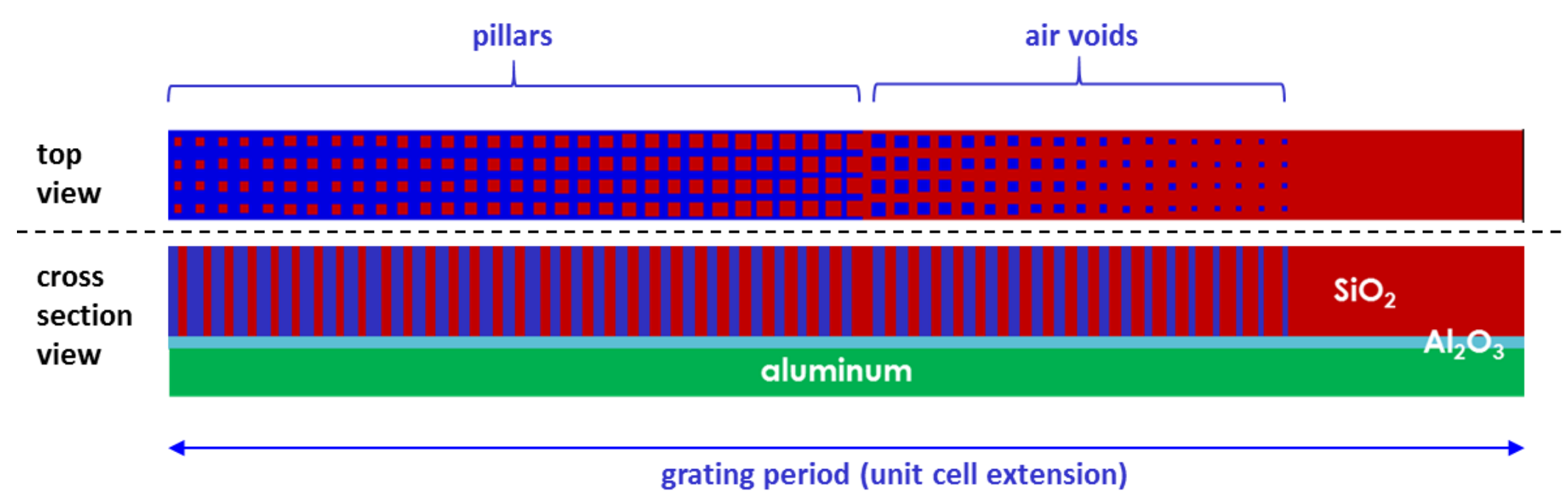

- smallest feature size: $120 \times 120 \mathrm{~nm}$

- depth: 550nm 


\section{Optical Performance}
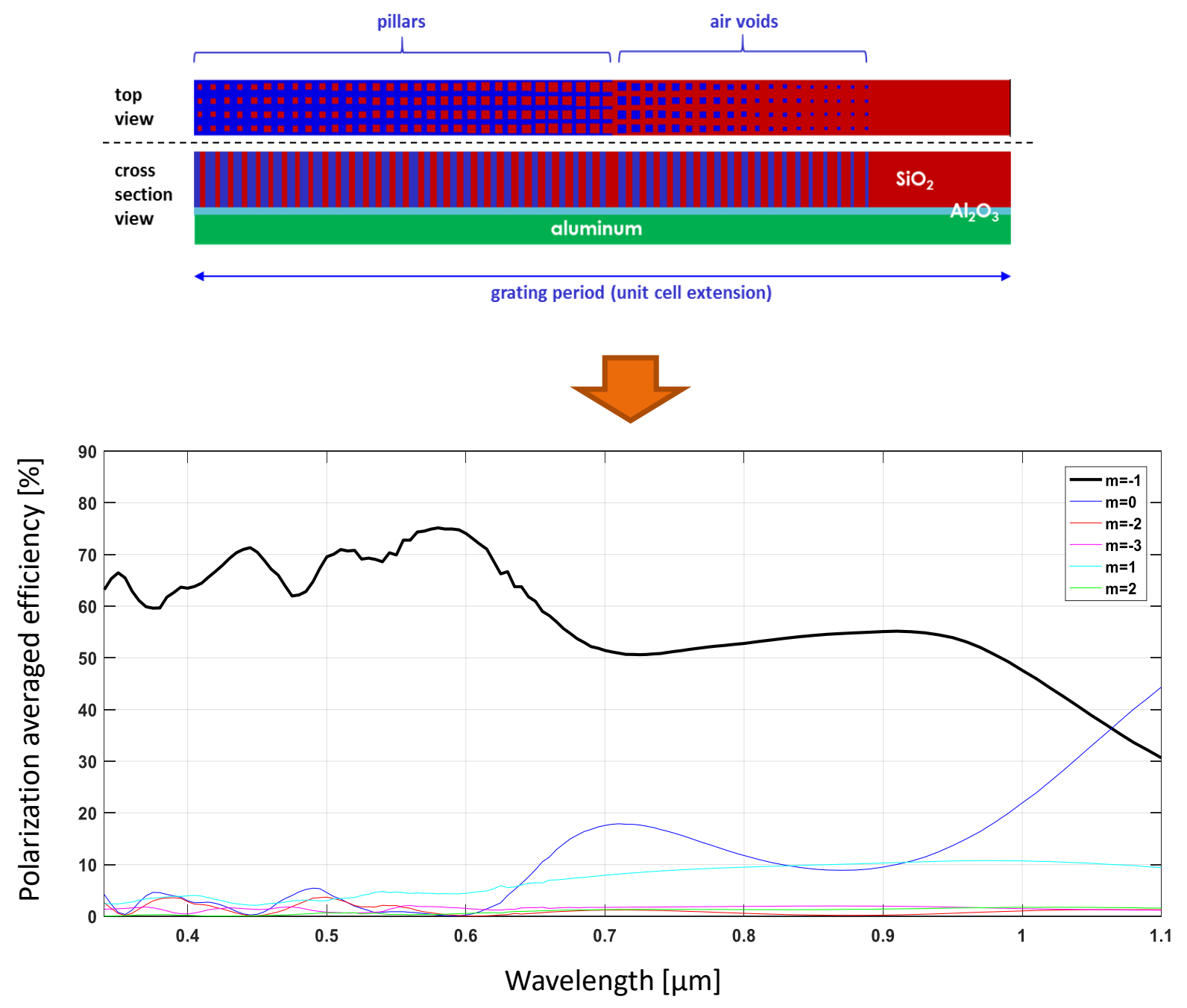


\section{Manufacturing via Electron Beam Lithography}

$>$ Grating Manufacturing on Plane Silicon Substrate

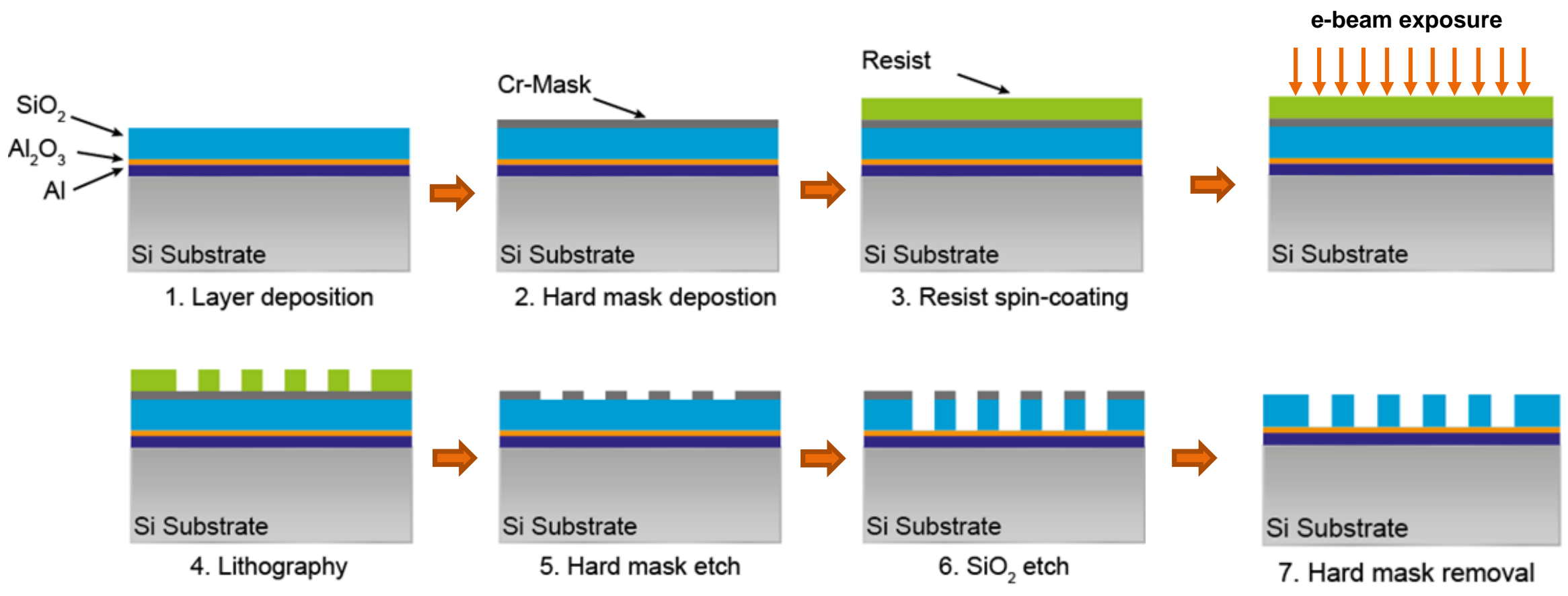




\section{Manufacturing via Electron Beam Lithography}

$>$ Grating Manufacturing on Plane Silicon Substrate

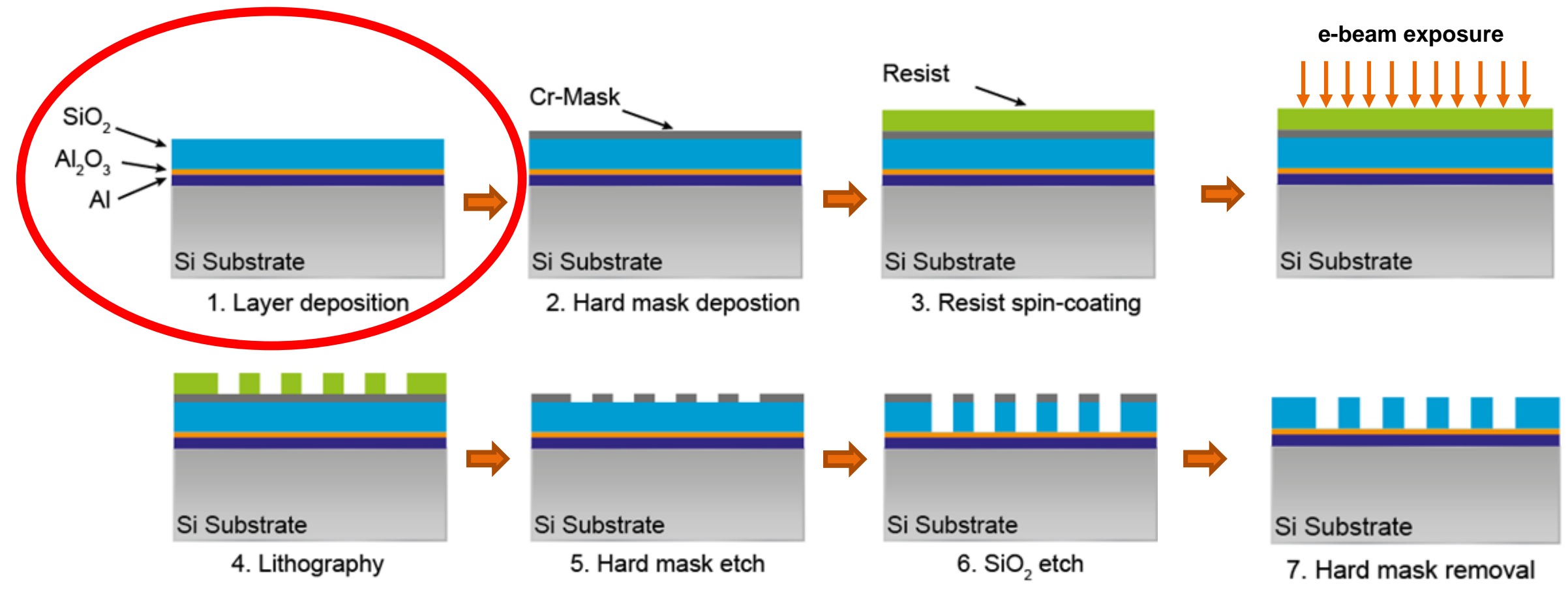

let's have a deeper look into some details ... 


\section{Aluminum Reflection Layer}

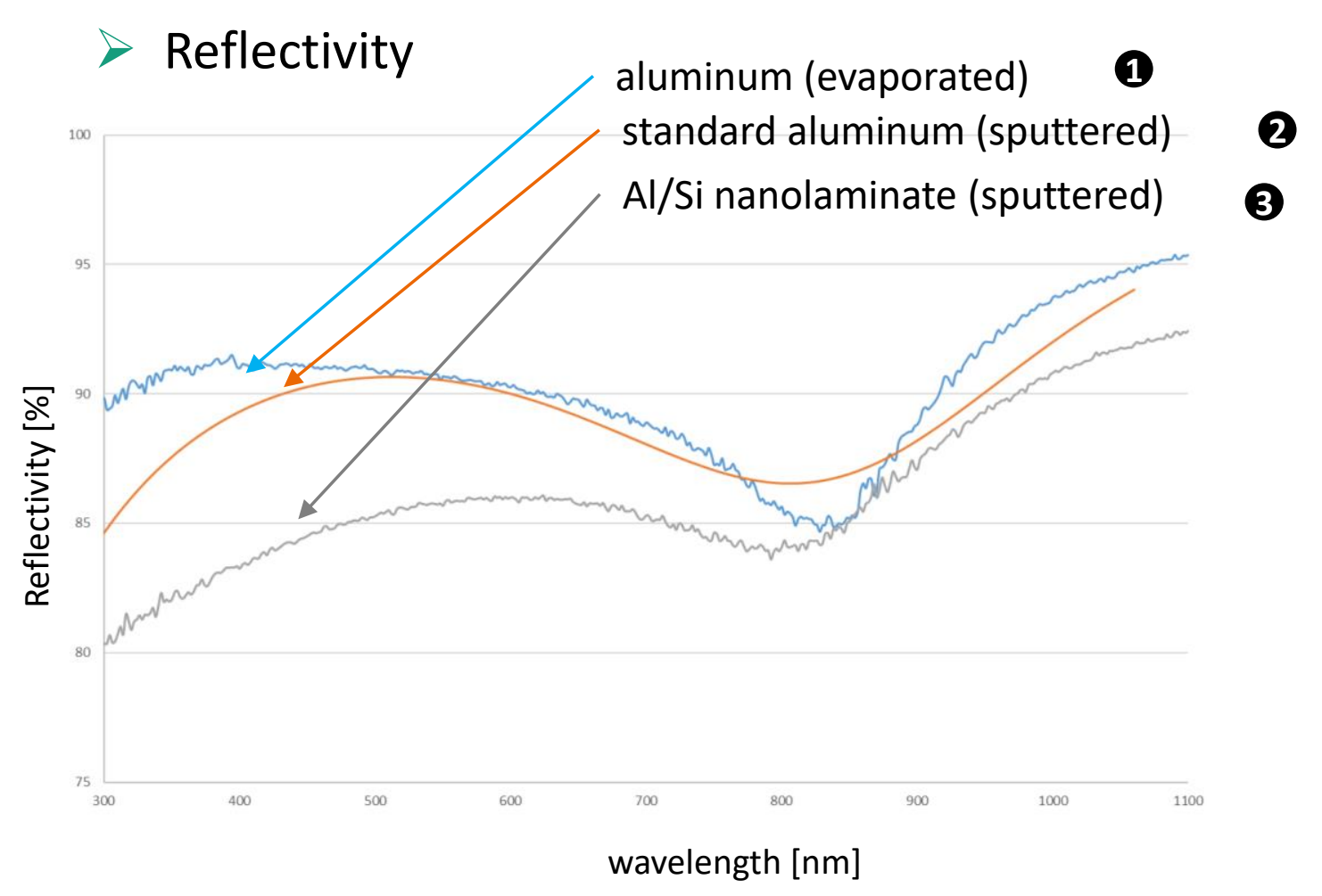




\section{Aluminum Reflection Layer}

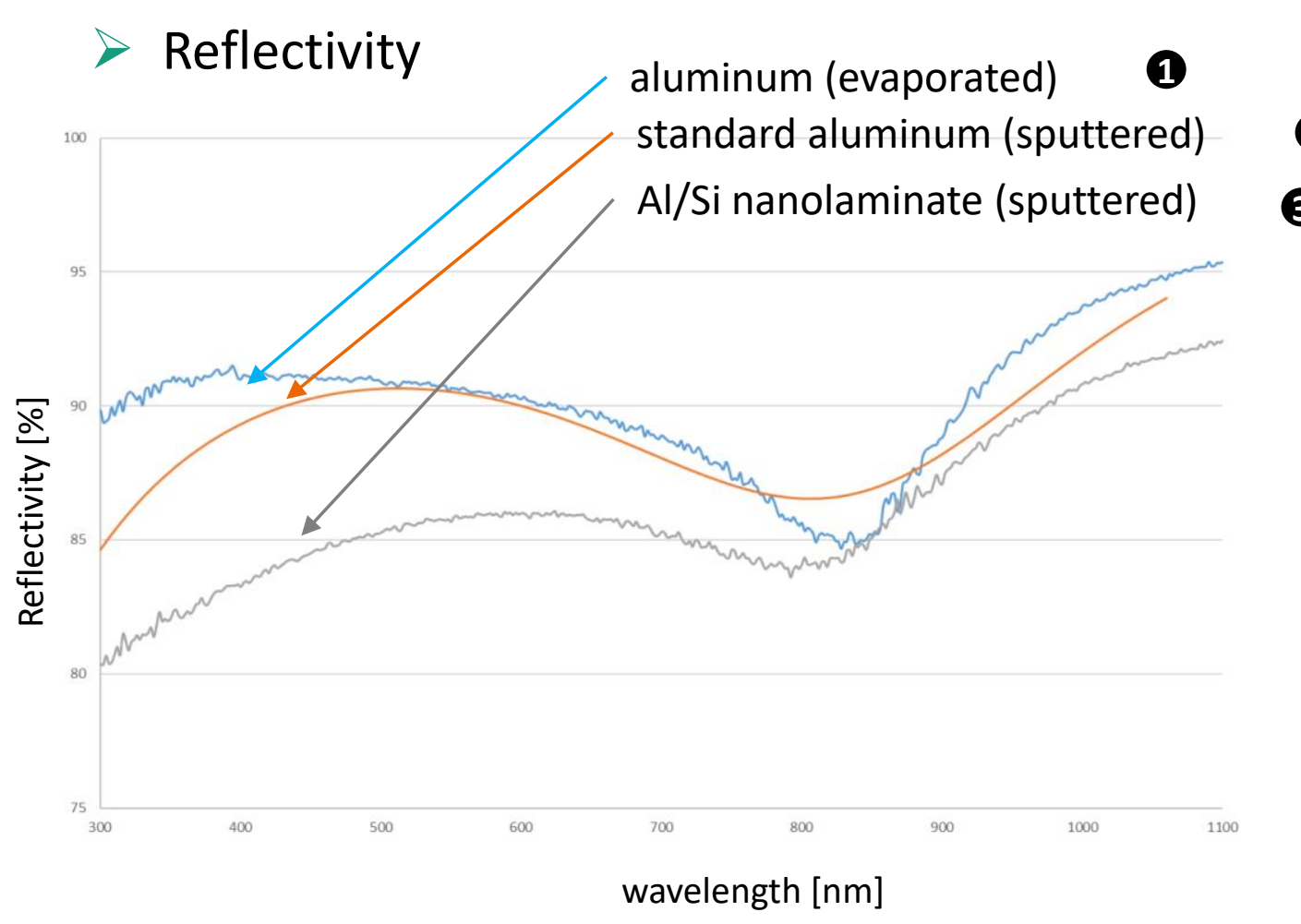

2

3

Surface Roughness

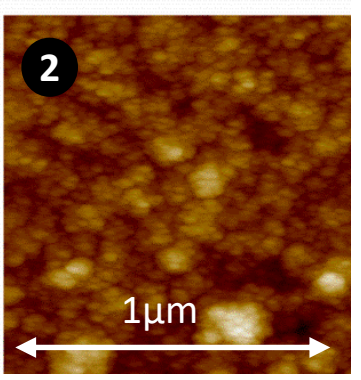

RMS 3.8nm

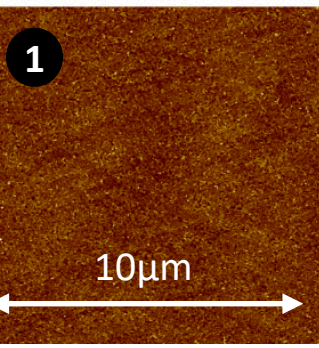

RMS 1.1nm

3

RMS 0.7nm

$10 \mu \mathrm{m}$ 


\section{Aluminum Reflection Layer}

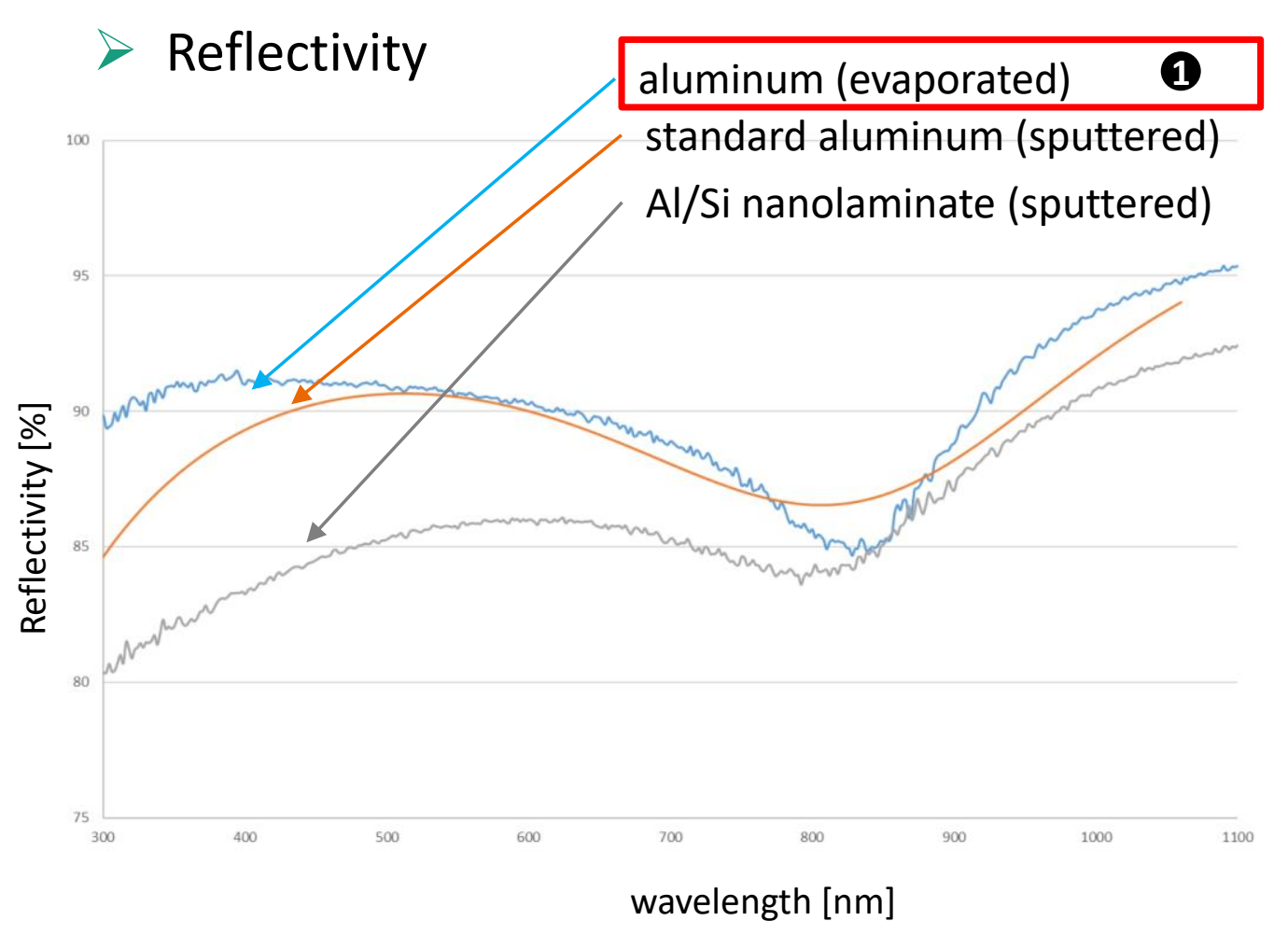

2

(3)

- use of evaporated aluminum for best compromise between surface roghness and reflectivity

Surface Roughness

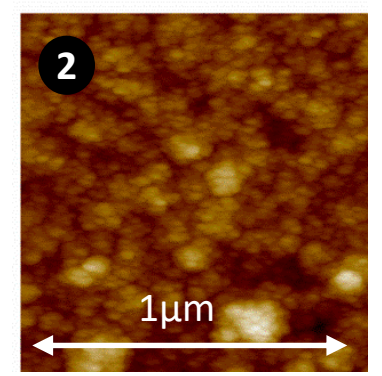

RMS 3.8nm

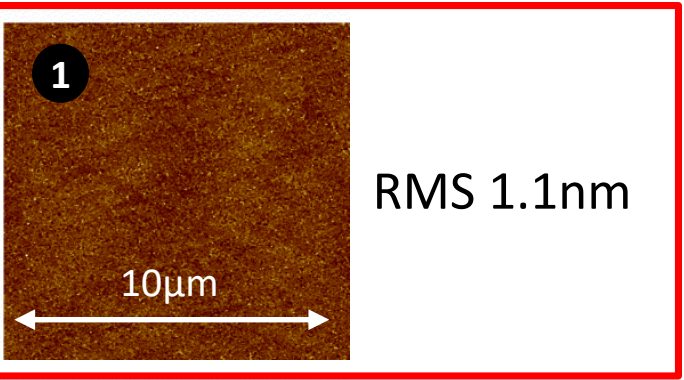

3

RMS $0.7 \mathrm{~nm}$ 


\section{FullSize WideBand Grating}

$>$ manufactured prototype

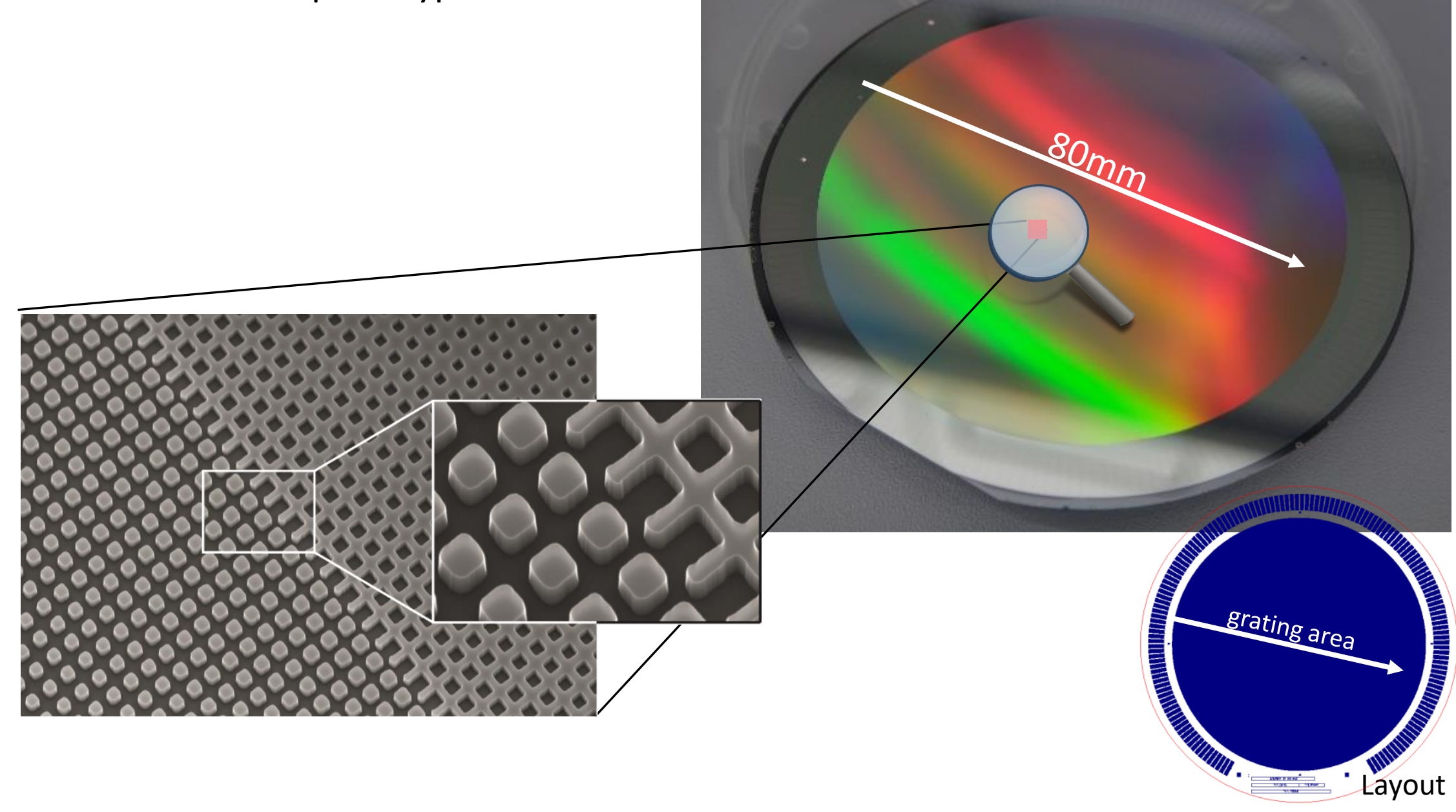




\section{Diffraction Efficiency}

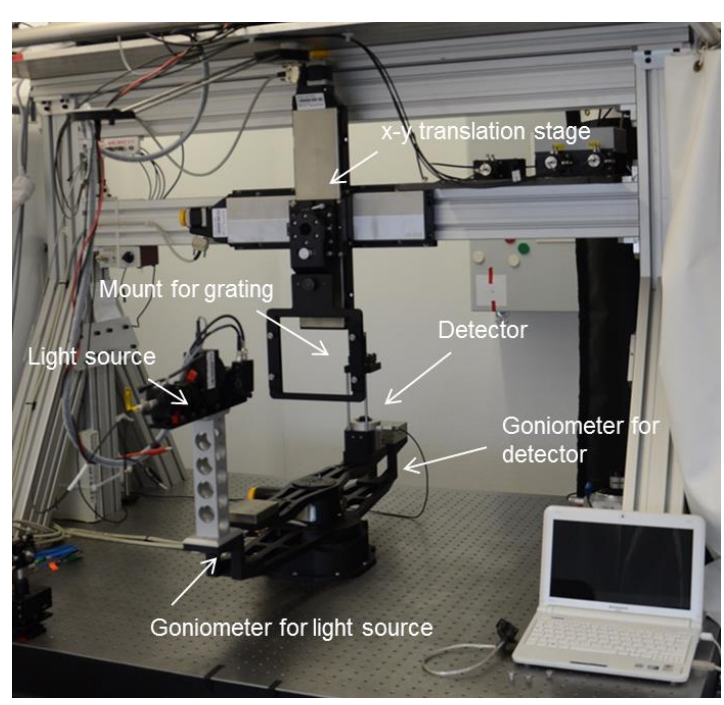

$>$ double-goniometer setup.

$>$ spatial mapping $(x / y)$

$>$ fiber-coupled sources

$>$ UV - SWIR2

$>$ monochromatic illumination

$>$ detection via integrating sphere
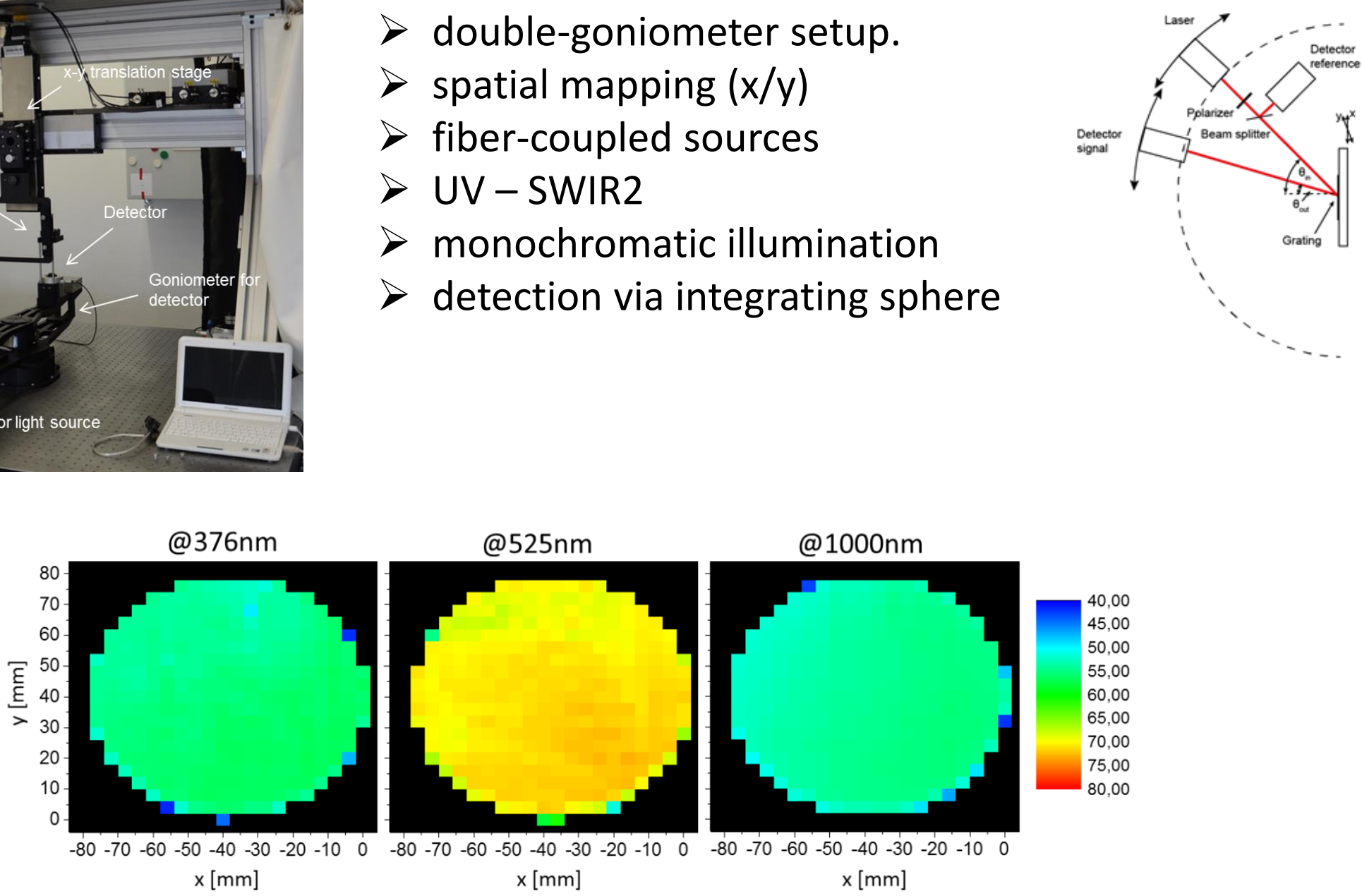


\section{Diffraction Efficiency}

> Spatially resolved efficiency

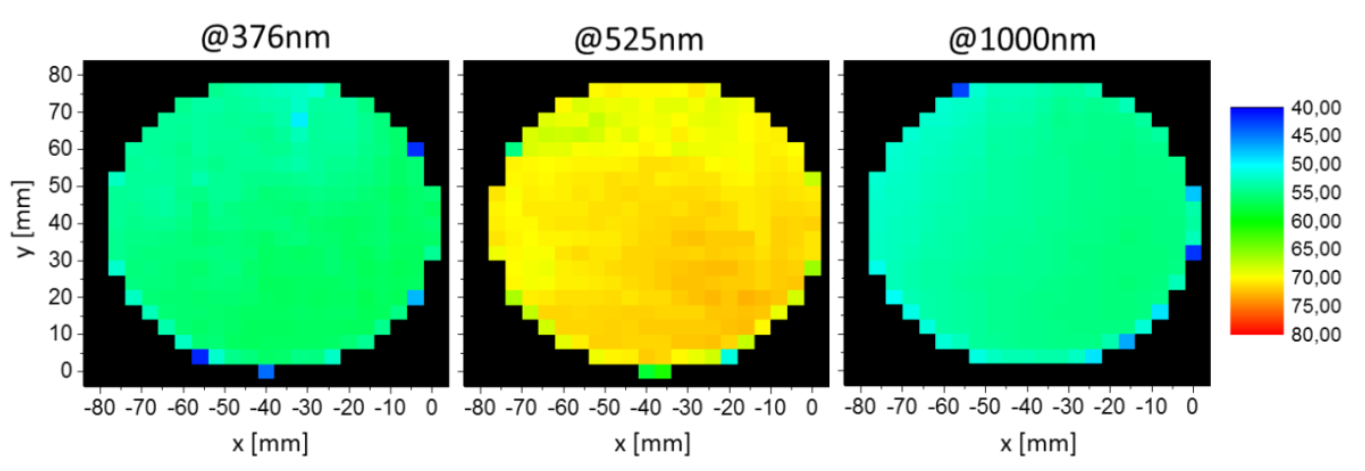

$>$ Spatial average throughout Grating Area

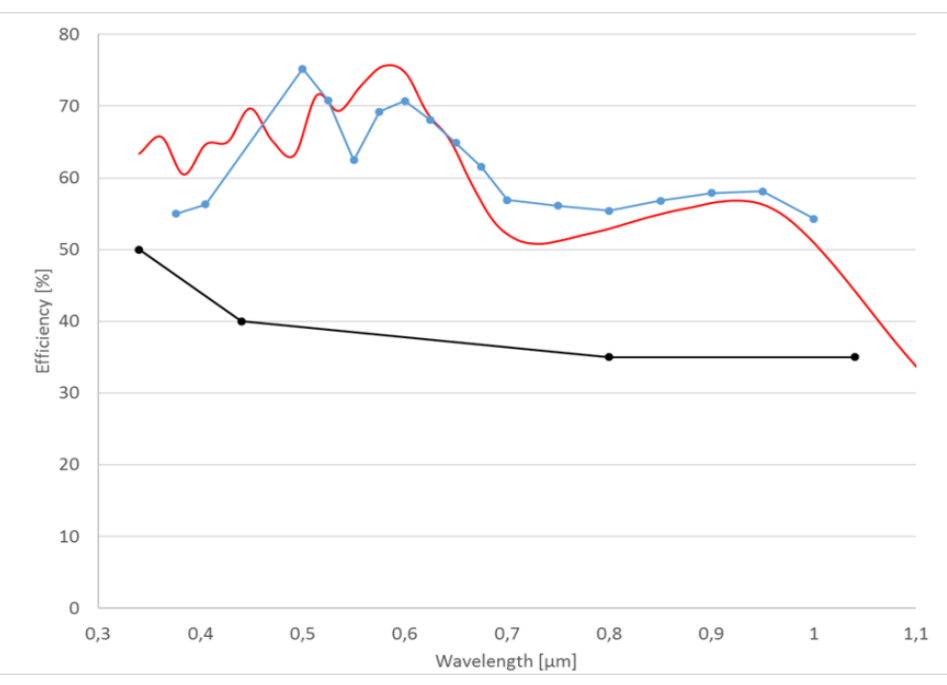

$\rightarrow$ measurement

design

requirement 


\section{Angle Resolved Scattering}

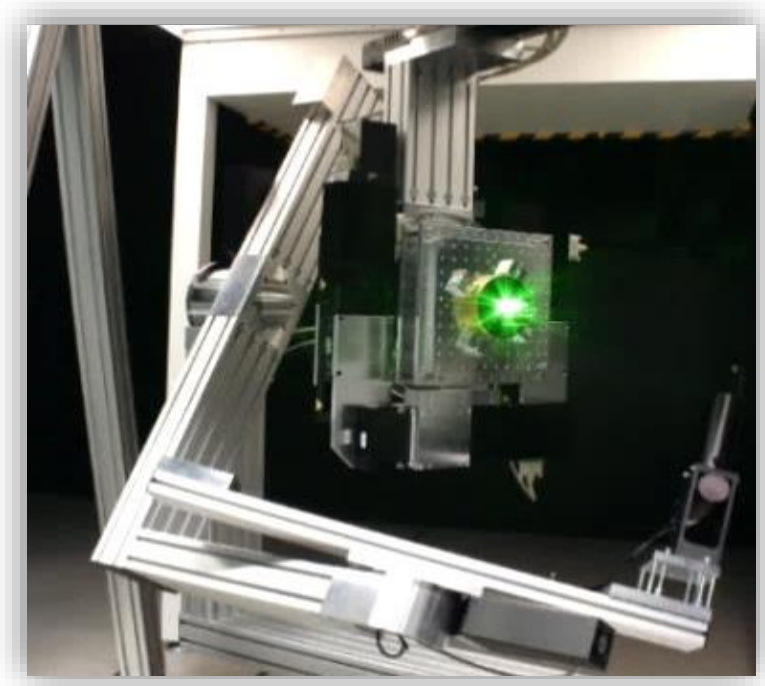

IOF ALBATROSS sample

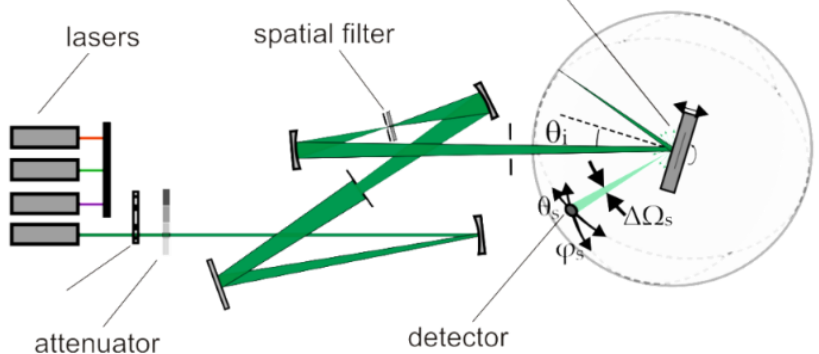

attenuator

detector

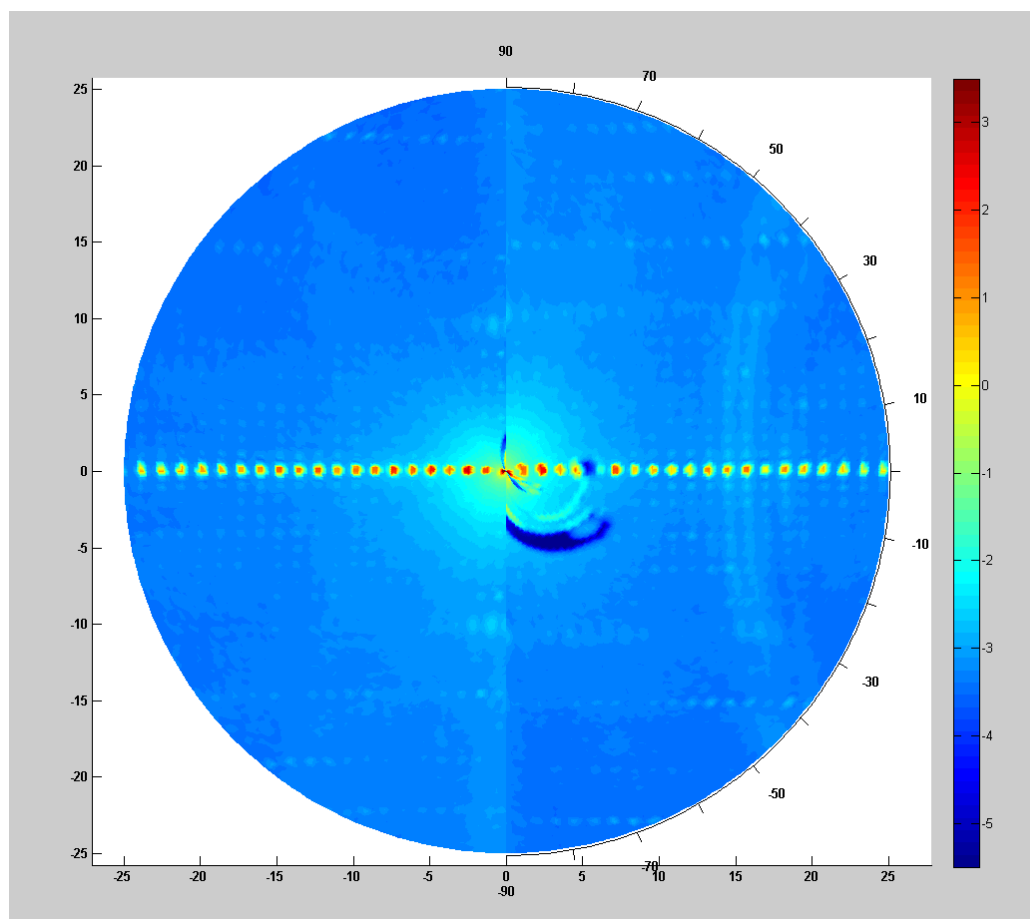




\section{Angle Resolved Scattering}

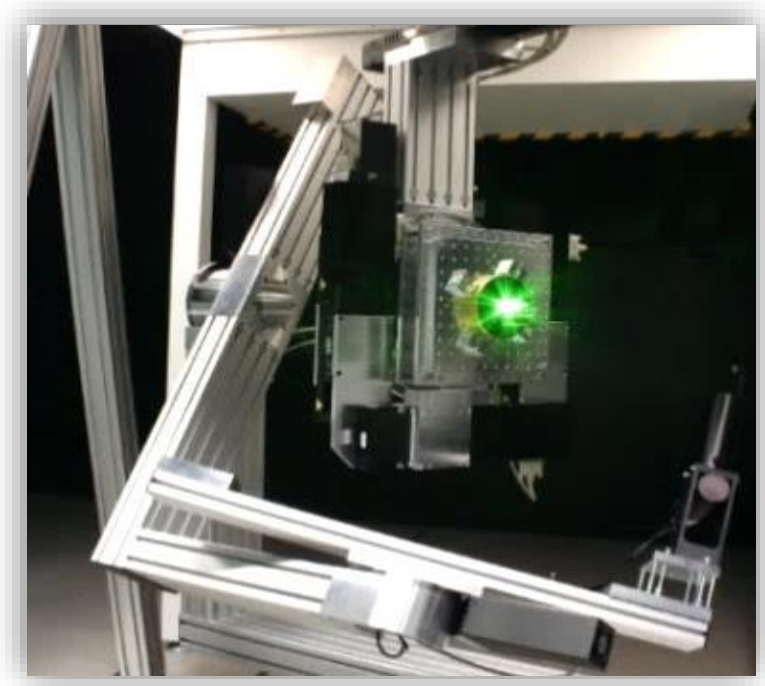

IOF ALBATROSS sample

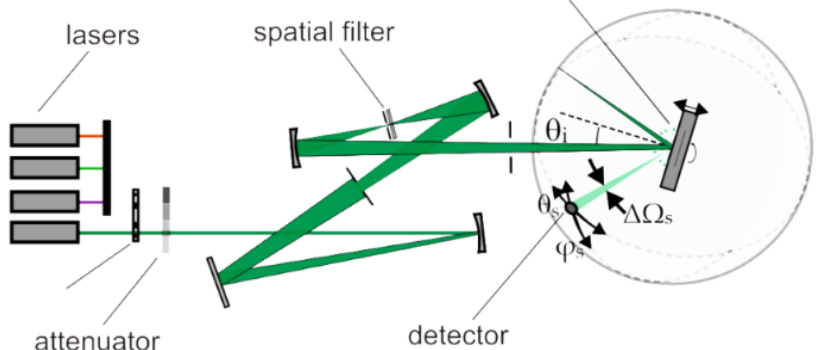

attenuator

detector

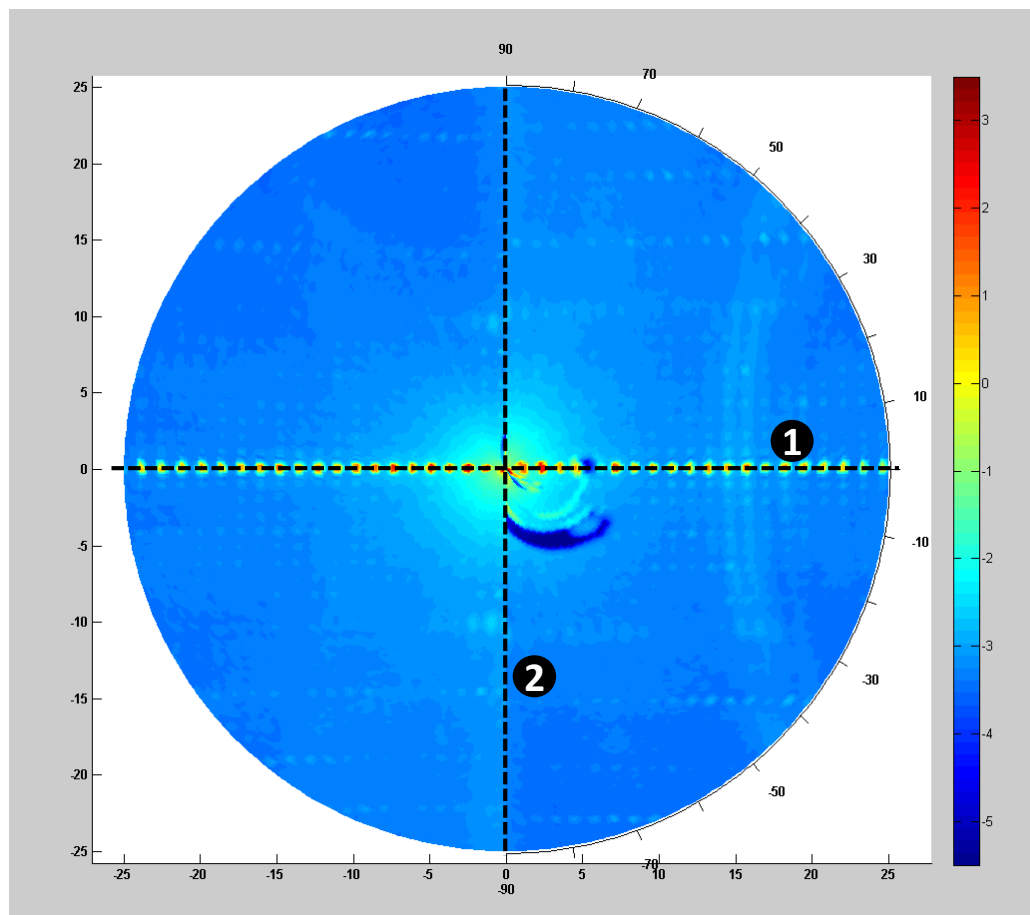




\section{Angle Resolved Scattering}

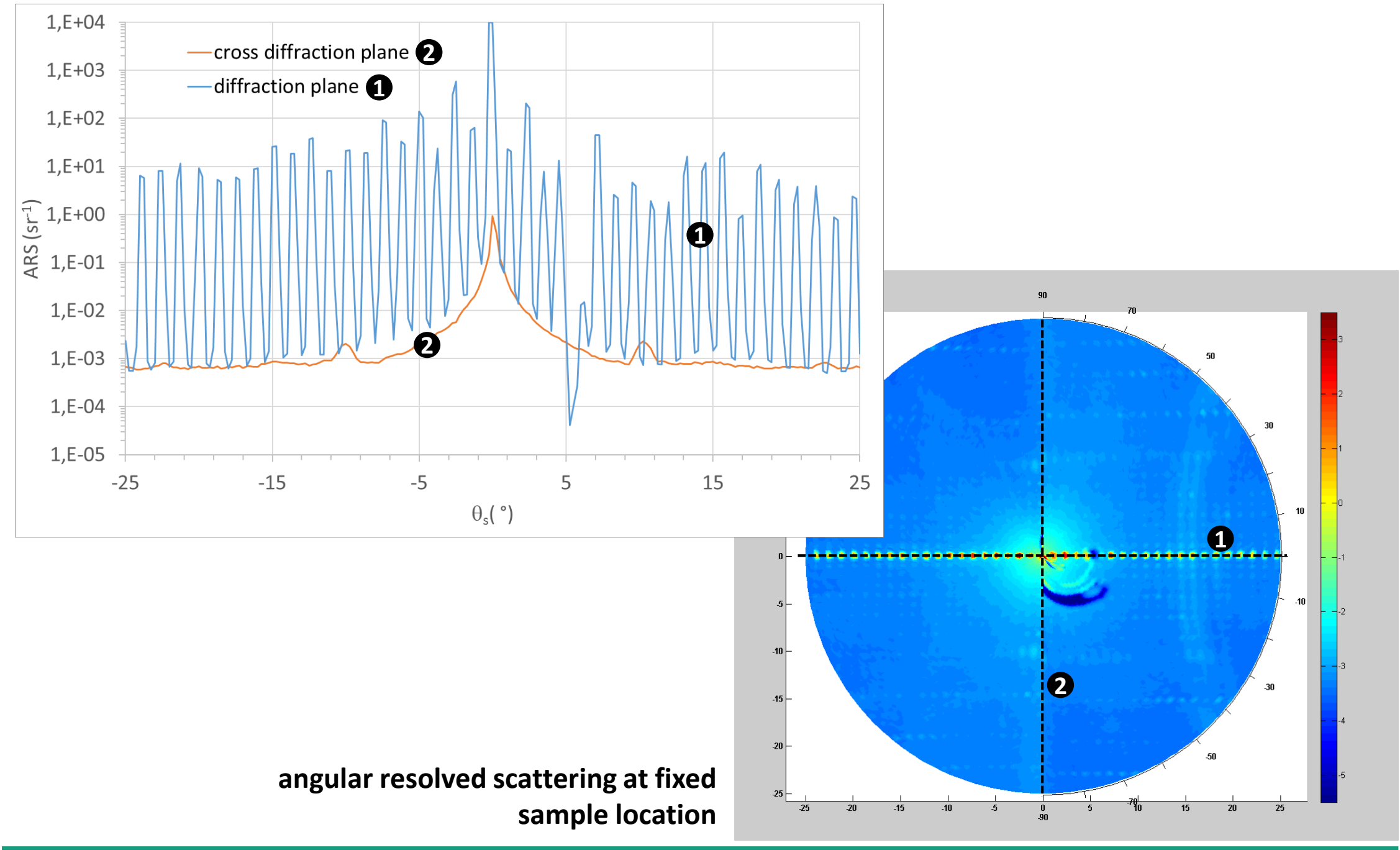




\section{Wavefront Error}

$>$ WaveFront Error induced by Lithography
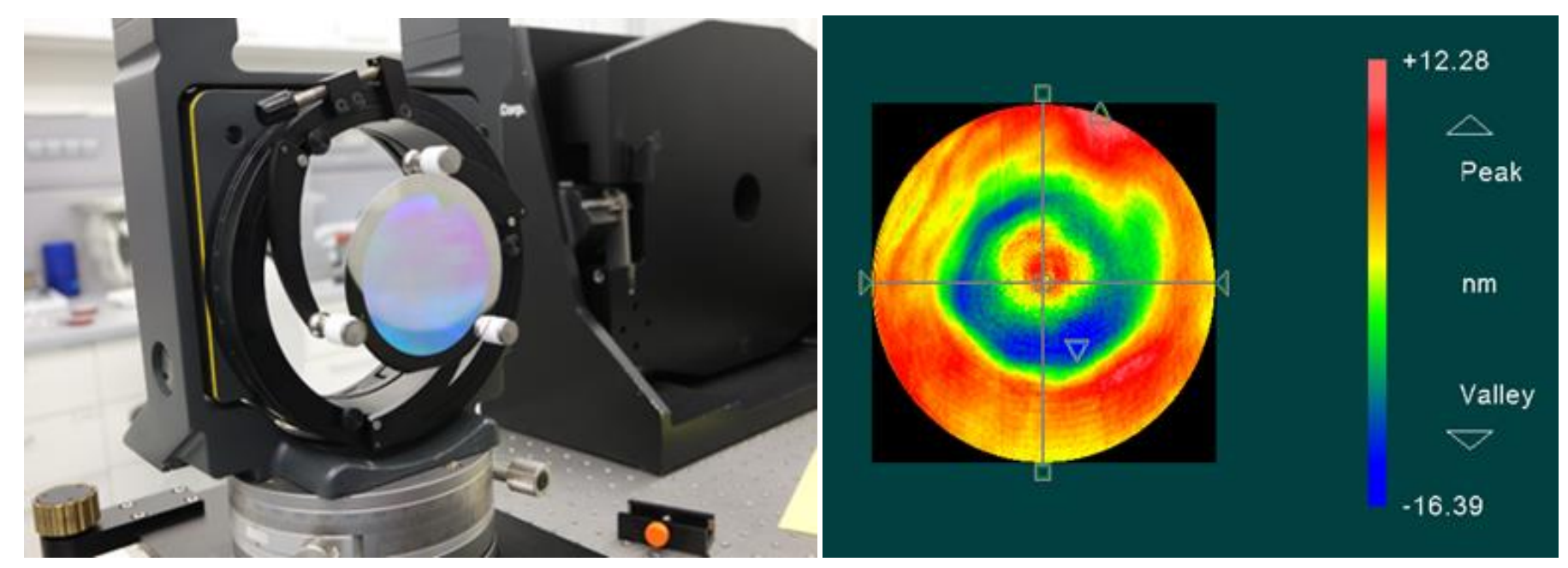

piston/tilt removed

- measurement in -1. and +1. diffraction order in two separate "shots"

- difference between -1 and +1 . removes influence of substrate deformation

WFE $<5.4 \mathrm{~nm}$ RMS (includes also measurement uncertainties) 


\section{Outlook: Extended Bandwidth}

$>$ Ultra-broadband operation: 340nm - 2500nm (UV - SWIR)

„Single“ - Binary Blazed grating working -1. diffraction order

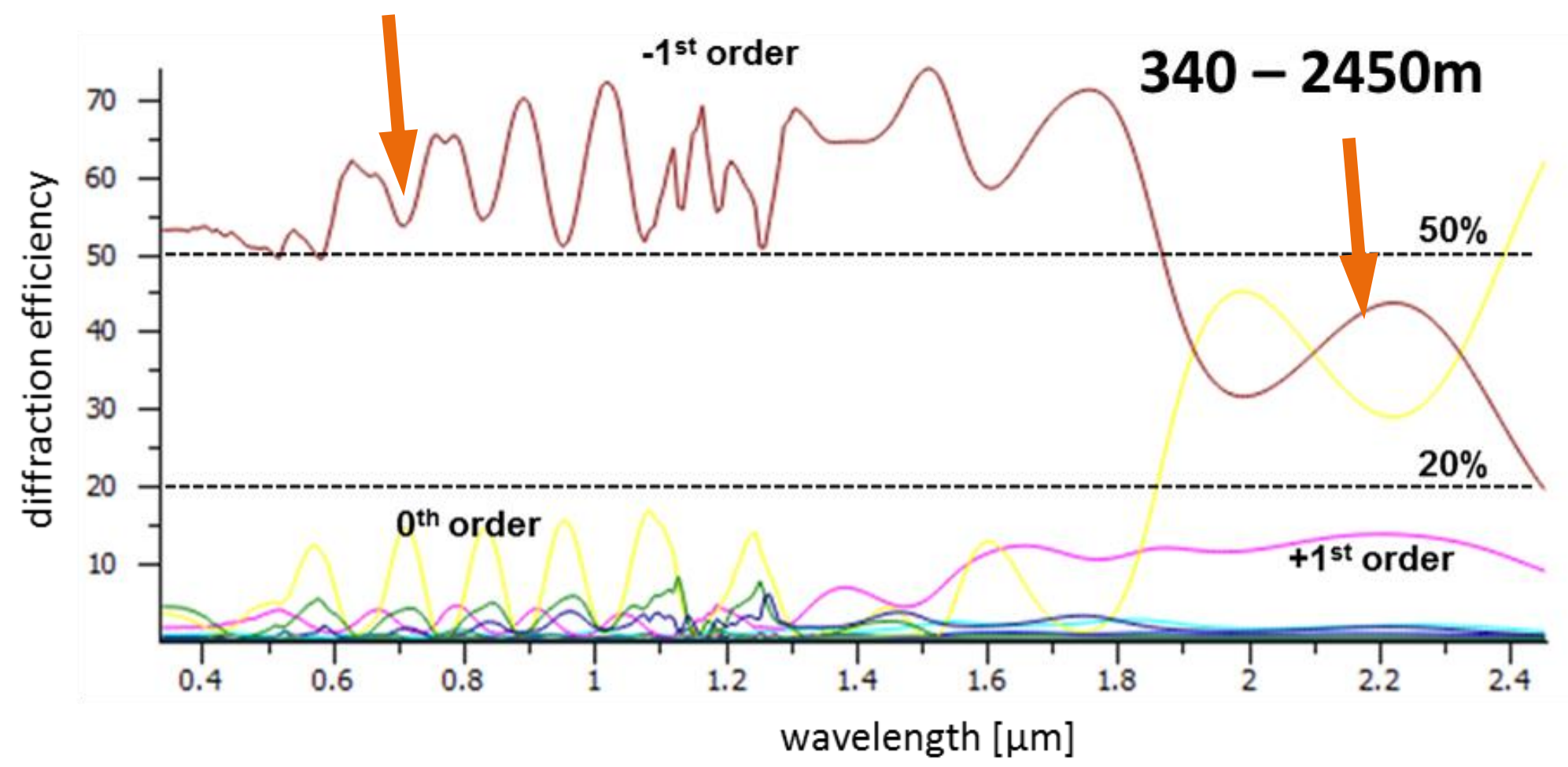




\section{Outlook: Extended Bandwidth}

$>$ Ultra-broadband operation: 340nm - 2500nm (UV - SWIR)

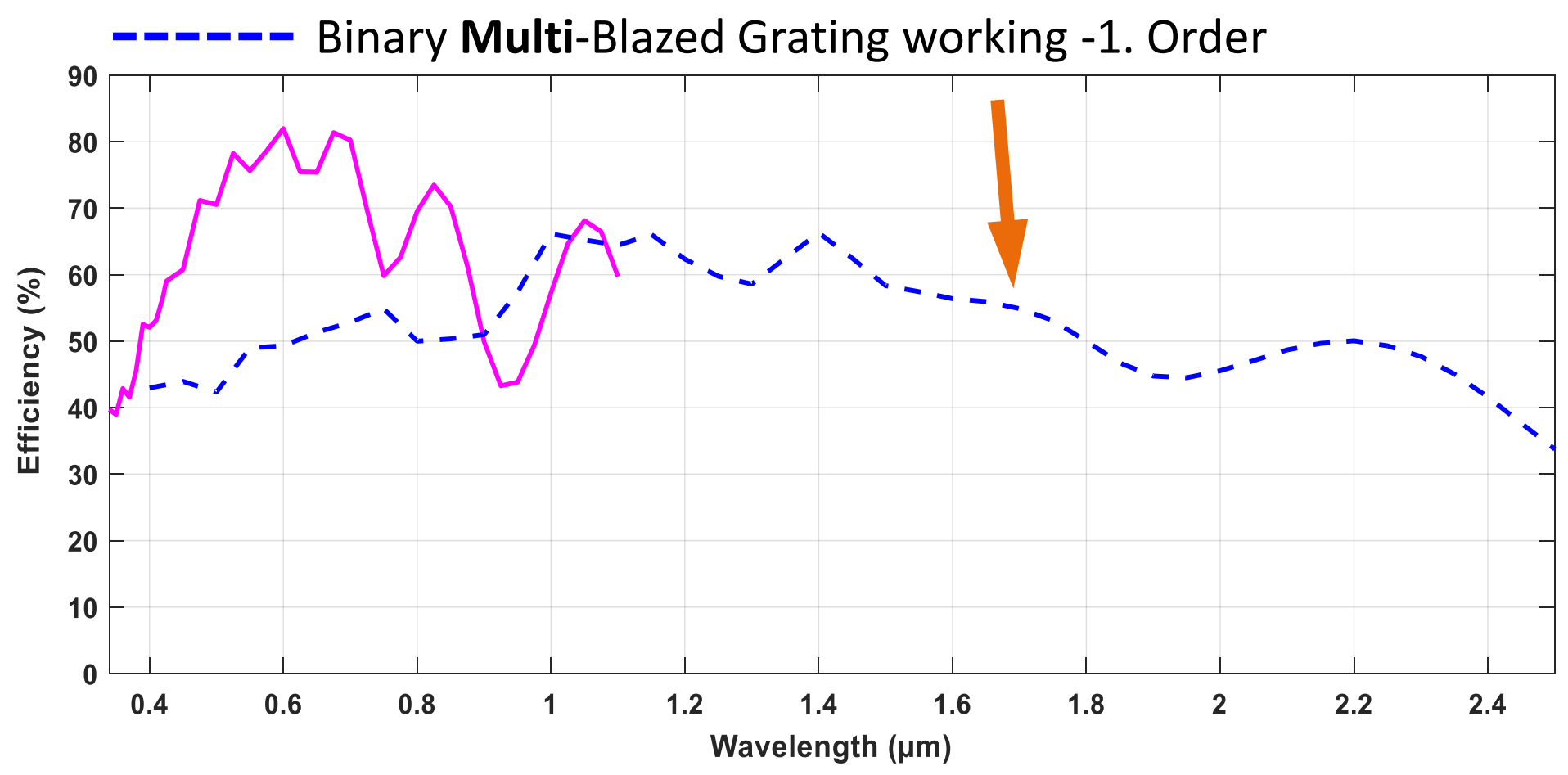

section \#1

section \#2 


\section{On the way to Curved Substrates - NIL approach (\#1)}

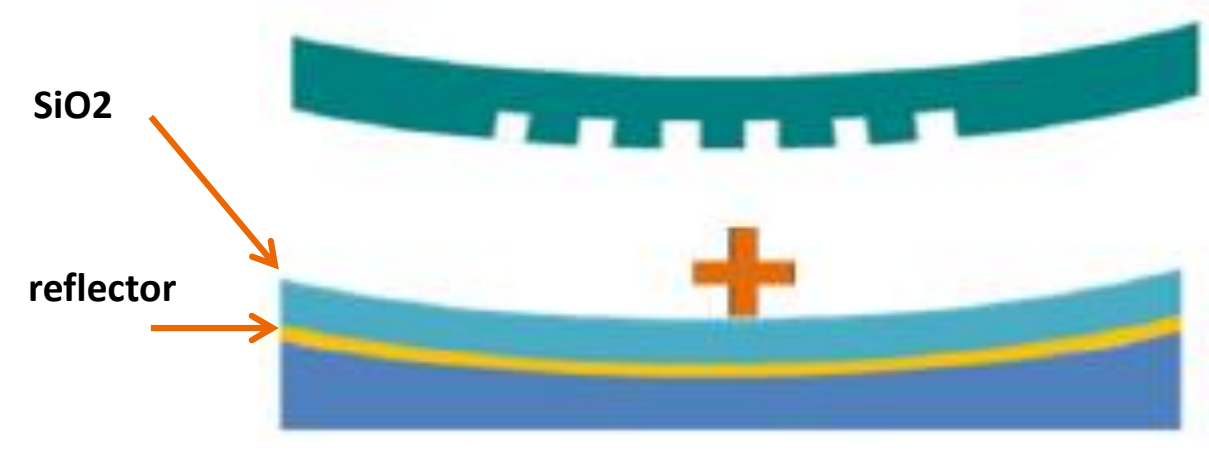

1. Soft stamp with grating pattern

2. Curved (grating) surface with Reflecting and SiO2 layer

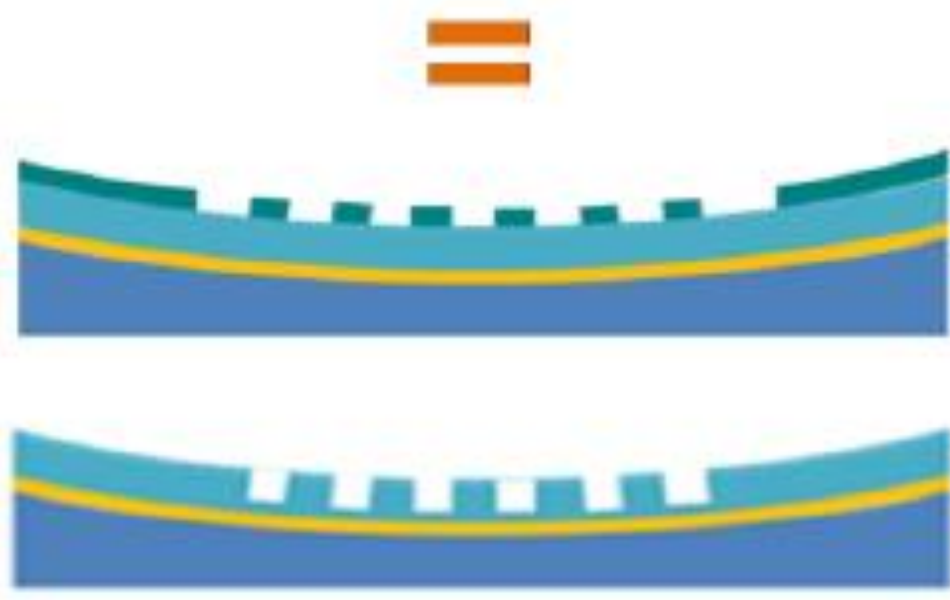

3. Imprint Litho + UV-curing

4. Transfer of the mask into $\mathrm{SiO} 2$ 


\section{On the way to Curved Substrates - NIL approach (\#1)}

$>$ NIL-lithography development for full-size plane substrates
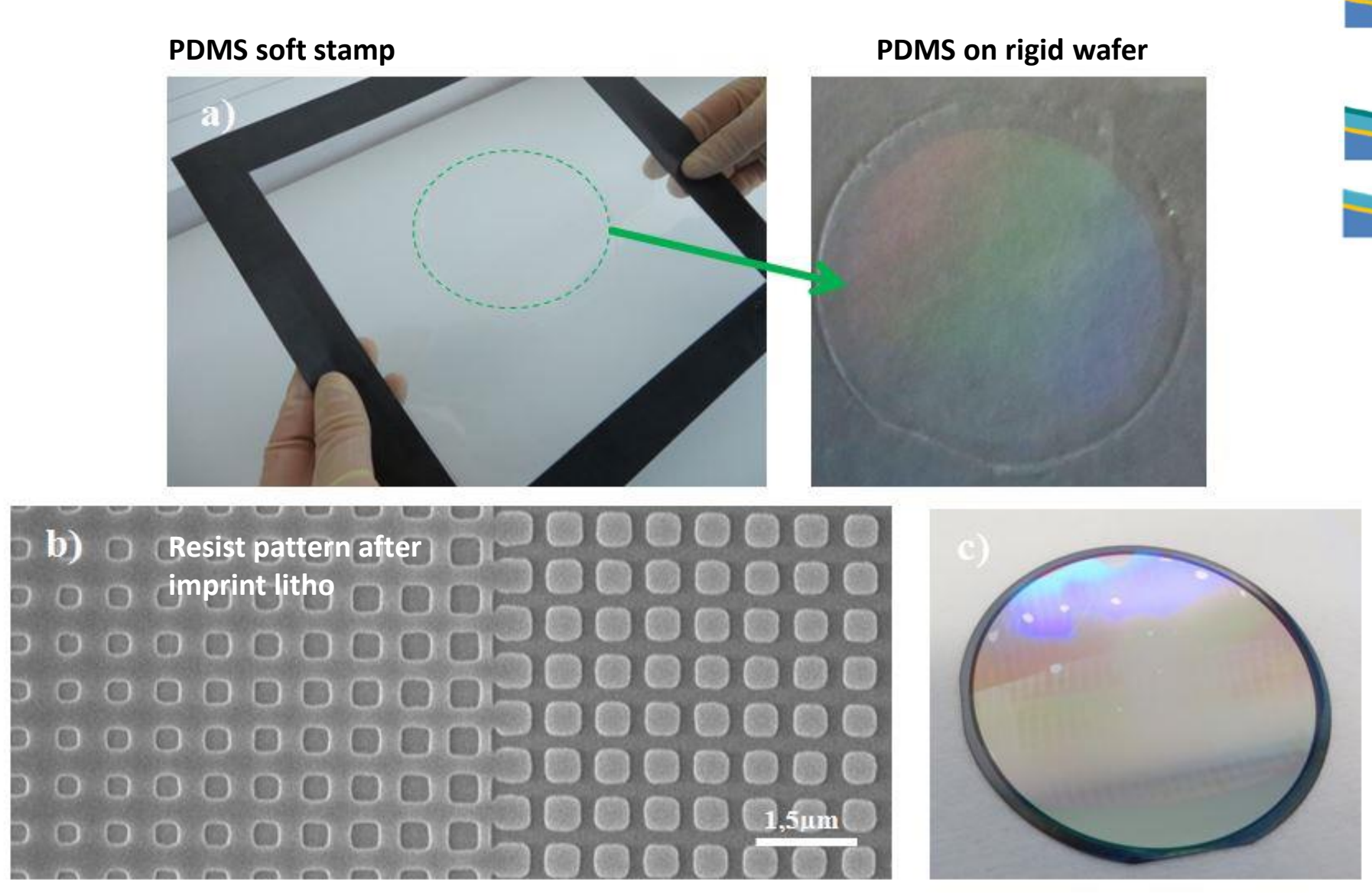

Final element after RIE etching of SiO2 


\section{On the way to Curved Substrates - bending appr. (\#2)}

$>$ Bending of a pre-manufactured grating into preform
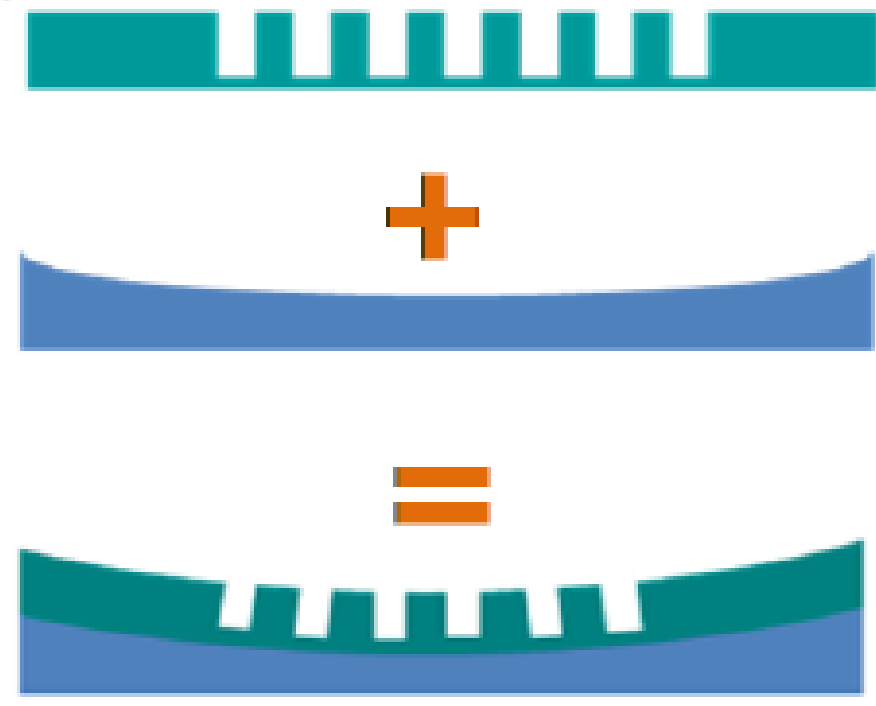

1. Grating on a thin silicon wafer

2. Preform: curved (grating) surface

3. Mechanically Deformation of the "grating wafer" to fit into the preform

$>$ Risks:

$>$ grating substrate must be thin $(<300 \mu \mathrm{m})$

$>$ radius of curvature is limited to "moderate“ values.

$>$ stress is induced and conserved. 


\section{On the way to Curved Substrates - bending appr. (\#2)}

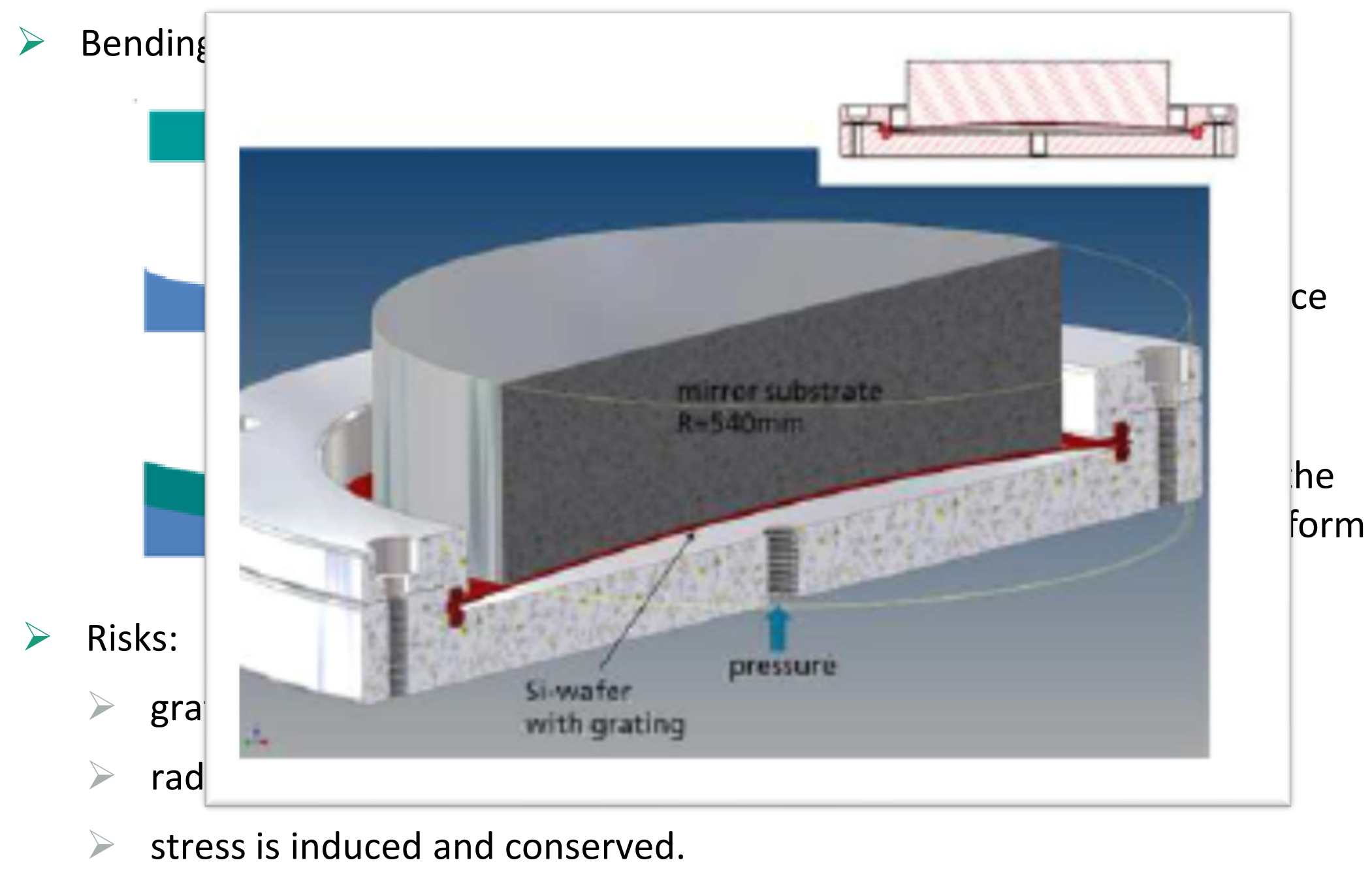




\section{Summary and Conclusion}

$>$ Broadband plane grating working between $340 \mathrm{~nm}-$ $1050 \mathrm{~nm}$ on an aperture of $80 \mathrm{~mm}$ was successfully demonstrated.

$>$ NIL Imprint master (in silicon) was also manufactured

$>$ Diffraction efficiency:

$>$ Peak: 75\%

$>$ Minimum: $50 \%-55 \%$

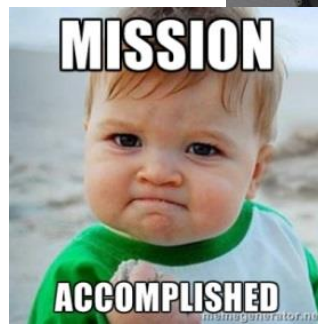

To Do...

$>$ Homogeneity of the NIL approach in 4-inch scale

$>$ Application to curved substrates (with 2 approached) 


\section{BackUp}




\section{Spatial Mapping of StrayLight}

> Scattered Light at fixed angular postion

$>$ Scan throughout Grating Area

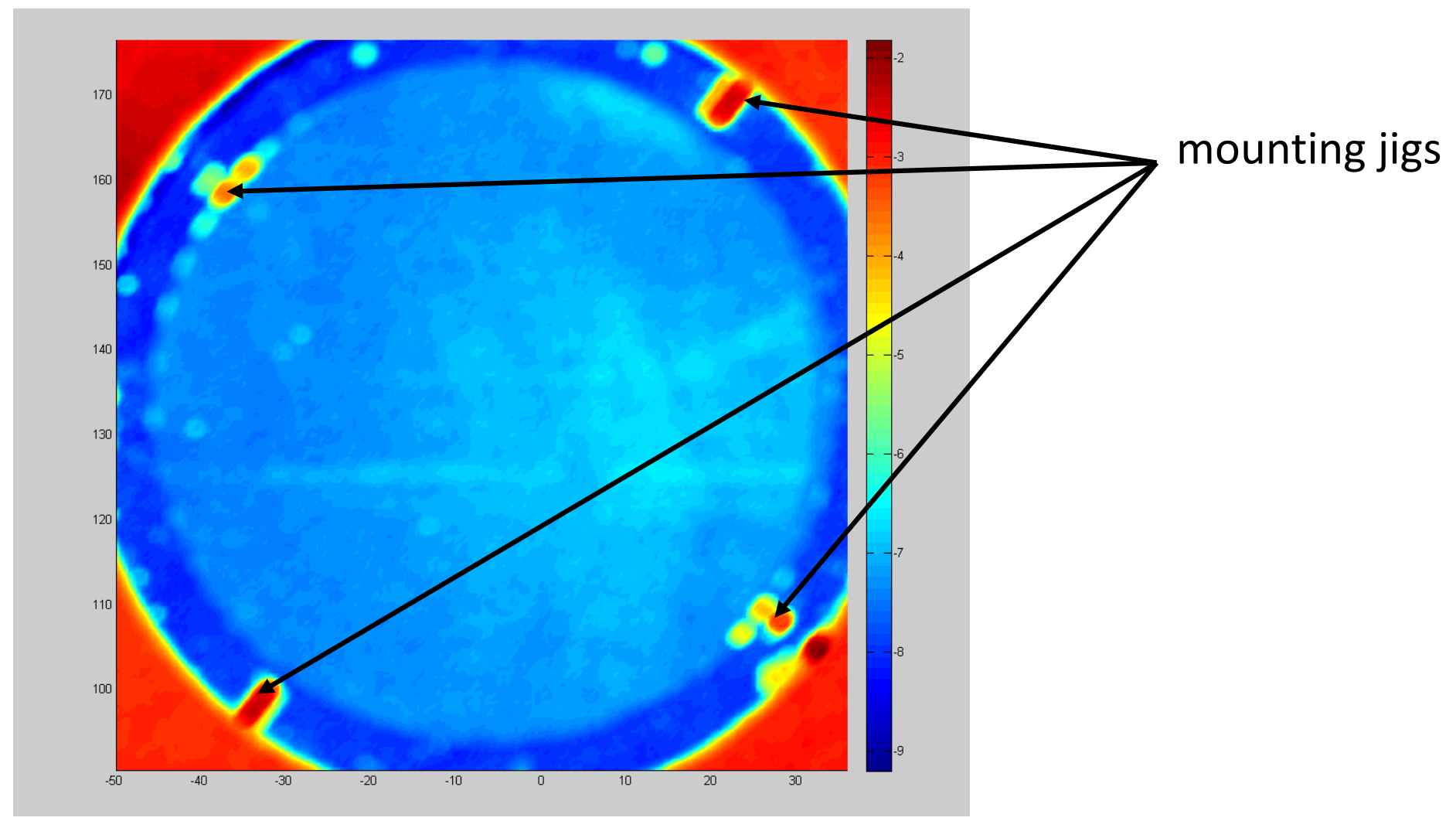

